\title{
ON SECANT DIMENSIONS AND IDENTIFIABILITY OF FLAG VARIETIES
}

\author{
AGEU BARBOSA FREIRE, ALEX CASAROTTI, AND ALEX MASSARENTI
}

\begin{abstract}
AвStract. We investigate the secant dimensions and the identifiablity of flag varieties parametrizing flag of sub vector spaces of a fixed vector space. We give numerical conditions ensuring that secant varieties of flag varieties have the expected dimension, and that a general point on these secant varieties is identifiable.
\end{abstract}

\section{Contents}

1. Introduction

2. Higher osculating behavior of products of Grassmannians

3. On secant defectivity of products of Grassmannians

4. On secant defectivity of flag varieties

5. On the chordal variety of $\mathbb{F}(0, k ; n)$

References

\section{INTRODUCTION}

In the most general contest, a flag variety is a projective variety homogeneous under a complex linear algebraic group. Flag varieties play a central role in algebraic geometry, combinatorics, and representation theory [Bri05, BL18].

Fix a vector space $V \cong \mathbb{C}^{n+1}$, over an algebraically closed field $K$ of characteristic zero, and integers $k_{1} \leq \ldots \leq k_{r}$. Let $\mathbb{G}\left(k_{i}, n\right) \subset \mathbb{P}^{N_{i}}$, where $N_{i}=\left(\begin{array}{c}n+1 \\ k_{i}+1\end{array}\right)-1$, be the Grassmannians of $k_{i}$-dimensional linear subspace of $\mathbb{P}(V)$ in its Plücker embedding. We have an embedding of the product of these Grassmannians

$$
\mathbb{G}\left(k_{1}, n\right) \times \cdots \times \mathbb{G}\left(k_{r}, n\right) \subset \mathbb{P}^{N_{1}} \times \cdots \times \mathbb{P}^{N_{r}} \subset \mathbb{P}^{N}
$$

where $N=\left(\begin{array}{c}n+1 \\ k_{1}+1\end{array}\right) \cdots\left(\begin{array}{c}n+1 \\ k_{r}+1\end{array}\right)-1$.

The flag variety $\mathbb{F}\left(k_{1}, \ldots, k_{r} ; n\right)$ is the set of flags, that is nested subspaces, $V_{k_{1}} \subset \cdots \subset V_{k_{r}} \subsetneq V$. This is a subvariety of the product of Grassmannian $\prod_{i=1}^{r} \mathbb{G}\left(k_{i}, n\right)$. Hence, via a product of Plücker embeddings followed by a Segre embedding we can embed $\mathbb{F}\left(k_{1}, \ldots, k_{r} ; n\right)$

$$
\mathbb{F}\left(k_{1}, \ldots, k_{r} ; n\right) \hookrightarrow \mathbb{P}^{N_{1}} \times \cdots \times \mathbb{P}^{N_{r}} \hookrightarrow \mathbb{P}^{N}
$$

Consider natural numbers $a_{1}, \ldots, a_{n}$ such that $a_{k_{1}+1}=\cdots=a_{k_{r}+1}=1$ and $a_{i}=0$ for all $i \notin\left\{k_{1}+1, \ldots, k_{r}+\right.$ $1\}$. Then, $\mathbb{F}\left(k_{1}, \ldots, k_{r} ; n\right)$ generates the subspace

$$
\mathbb{P}\left(\Gamma_{a_{1}, \ldots, a_{n}}\right) \subseteq \mathbb{P}\left(\bigwedge^{k_{1}+1} V \otimes \cdots \otimes \bigwedge^{k_{r}+1} V\right) \subseteq \mathbb{P}^{N}
$$

where $\Gamma_{a_{1}, \ldots, a_{n}}$ is the irreducible representation of $\mathfrak{s l}_{n+1} \mathbb{C}$ with highest weight $\left(a_{1}+\cdots+a_{n}\right) L_{1}+\cdots+a_{n} L_{n}$, and $L_{1}+\cdots+L_{k}$ is the highest weight of the irreducible representation $\bigwedge^{k} V$. We will denote $\Gamma_{a_{1}, \ldots, a_{n}}$ simply by $\Gamma_{a}$. By the Weyl character formula we have that

$$
\operatorname{dim} \mathbb{P}\left(\Gamma_{a}\right)=\prod_{1 \leq i<j \leq n+1} \frac{\left(a_{i}+\cdots+a_{j-1}\right)+j-i}{j-i}-1
$$

Furthermore, $\operatorname{dim} \mathbb{F}\left(k_{1}, \ldots, k_{r} ; n\right)=\left(k_{1}+1\right)\left(n-k_{1}\right)+\sum_{j=2}^{i}\left(n-k_{j}\right)\left(k_{j}-k_{j-1}\right)$ and $\mathbb{F}\left(k_{1}, \ldots, k_{r} ; n\right)=\mathbb{P}\left(\Gamma_{a}\right) \cap$ $\prod_{i=1}^{r} \mathbb{G}\left(k_{i}, n\right) \subset \mathbb{P}^{N}$.

Date: December 3, 2019.

2010 Mathematics Subject Classification. Primary 14N05, 14N15, 14M15; Secondary 14E05, 15 A69, 15 A75.

Key words and phrases. Flag varieties, secant varieties, identifiability. 
The geometry of these varieties has been investigated mostly from the point of view of Schubert calculus Bri05 and dual defectivity Tev05. Secant varieties of small dimensional flag varieties have been studied in BD10 by taking advantage of the tropical approach to secant dimensions introduced by J. Draisma in Dra08.

The $h$-secant variety $\operatorname{Sec}_{h}(X)$ of a non-degenerate $n$-dimensional variety $X \subset \mathbb{P}^{N}$ is the Zariski closure of the union of all linear spaces spanned by collections of $h$ points of $X$. The expected dimension of $\mathbb{S e c}_{h}(X)$ is $\operatorname{expdim}\left(\operatorname{Sec}_{h}(X)\right):=\min \{n h+h-1, N\}$. In general, the actual dimension of $\operatorname{Sec}_{h}(X)$ may be smaller than the expected one. In this case, following [CC10, Section 2] we say that $X$ is $h$-defective and the number $\delta_{h}(X)=\operatorname{expdim}\left(\operatorname{Sec}_{h}(X)\right)-\operatorname{dim}\left(\operatorname{Sec}_{h}(X)\right)$ is called the $h$-secant defect of $X$.

We investigate secant defectivity of flag varieties following the machinery introduced in [MR19], which we now outline. Given general points $x_{1}, \ldots, x_{h} \in X \subset \mathbb{P}^{N}$, consider the linear projection $\tau_{X, h}: X \subseteq \mathbb{P}^{N}-\rightarrow \mathbb{P}^{N_{h}}$, with center $\left\langle T_{x_{1}} X, \ldots, T_{x_{h}} X\right\rangle$, where $N_{h}:=N-1-\operatorname{dim}\left(\left\langle T_{x_{1}} X, \ldots, T_{x_{h}} X\right\rangle\right)$. [CC02, Proposition 3.5] yields that if $\tau_{X, h}$ is generically finite then $X$ is not $(h+1)$-defective. Given $p_{1}, \ldots, p_{l} \in X$ general points, we consider the linear projection $\Pi_{T_{p_{1}, \ldots, p_{l}}^{k_{1}, \ldots, k_{l}}}: X \subset \mathbb{P}^{N} \rightarrow \mathbb{P}^{N_{k_{1}}, \ldots, k_{l}}$ with center the $\operatorname{span}\left\langle T_{p_{1}}^{k_{1}} X, \ldots, T_{p_{l}}^{k_{l}} X\right\rangle$ of higher order osculating spaces. We can degenerate, under suitable conditions, the linear span of several tangent spaces $T_{x_{i}} X$ into a subspace contained in a single osculating space $T_{p}^{k} X$. So the tangential projection $\tau_{X, h}$ degenerates to a linear projection with center contained in $\left\langle T_{p_{1}}^{k_{1}} X, \ldots, T_{p_{l}}^{k_{l}} X\right\rangle$. If $\Pi_{T_{p_{1}, \ldots, p_{l}}^{k_{1}, \ldots, k_{l}}}$ is generically finite, then $\tau_{X, h}$ is generically finite as well, and we conclude that $X$ is not $(h+1)$-defective. In this paper we apply this strategy to flag varieties. We would like to stress that this approach, as the one introduced in [Dra08, depends heavily on an explicit parametrization of $X$. This method was successfully applied to other classes of homogeneous varieties such as Grassmannians MR19, Segre-Veronese varieties AMR19, Lagrangian Grassmannians and Spinor varieties FMR18. However, its application to flag varieties involves much more difficult computations compared with the case of the Grassmannians, this is particularly reflected in Section 4 where we introduce submersions of flag varieties into product of Grassmannians in order to study the relation among their higher osculating spaces.

Furthermore, our results on secant defectivity, combined with a recent result in CM19, allow us to produce a bound for identifiability of flag varieties. Recall that, given a non-degenerated variety $X \subset \mathbb{P}^{N}$, we say that a point $p \in \mathbb{P}^{N}$ is $h$-identifiable if it lies on a unique $(h-1)$-plane in $\mathbb{P}^{N}$ that is $h$-secant to $X$. Especially when $\mathbb{P}^{N}$ can be interpreted as a tensor space, identifiablity and tensor decomposition algorithms are central in applications for instance in biology, Blind Signal Separation, data compression algorithms, analysis of mixture models psycho-metrics, chemometrics, signal processing, numerical linear algebra, computer vision, numerical analysis, neuroscience and graph analysis DL13a, DL13b, DL15, KAL11, SB00, BK09, CGLM08, [LO15, MR13. Our main results in Theorem 4.14 and Corollary 4.15 can be summarized in the following statement.

Theorem 1.1. Consider a flag variety $\mathbb{F}\left(k_{1}, \ldots, k_{r} ; n\right)$. Assume that $n \geq 2 k_{j}+1$ for some index $j$ and let $l$ be the maximum among these $j$ 's. Then, for

$$
h \leq\left(\frac{n+1}{k_{l}+1}\right)^{\left\lfloor\log _{2}\left(\sum_{j=1}^{l} k_{j}+l-1\right)\right\rfloor}
$$

$\mathbb{F}\left(k_{1}, \ldots, k_{r} ; n\right)$ is not $(h+1)$-defective. Furthermore, under the same bound, the general point of the $h$-secant variety of $\mathbb{F}\left(k_{1}, \ldots, k_{r} ; n\right)$ is $h$-identifiable.

The paper is organized as follows: in Section 2 we study higher order osculating spaces of products of Grassmannians and the linear projections from them, in Section 3 we apply the method introduced in MR19] to products of Grassmannians, in Section 4 we get bounds for non-secant defectivity and identifiablity of flag varieties, and in Section 5 we investigate the variety of secant lines of spacial flag varieties of type $\mathbb{F}(0, k ; n)$.

Acknowledgments. The first named author would like to thank FAPERJ and Massimiliano Mella (PRIN 2015, Geometry of Algebraic Varieties, 2015EYPTSB-005) for the financial support, and the University of Ferrara for the hospitality during the period in which the majority of this work was completed. The third named author is a member of the Gruppo Nazionale per le Strutture Algebriche, Geometriche e le loro Applicazioni of the Istituto Nazionale di Alta Matematica F. Severi (GNSAGA-INDAM).

\section{Higher osculating Behavior of products of Grassmannians}

Consider the product $\mathbb{G}\left(k_{1}, n\right) \times \cdots \times \mathbb{G}\left(k_{r}, n\right) \subset \mathbb{P}^{N_{1}} \times \cdots \times \mathbb{P}^{N_{r}} \subset \mathbb{P}^{N}$, and given a non-negative integer $k$ define

$$
\Lambda_{k}=\{I \subset\{0, \ldots, n\}|| I \mid=k+1\}
$$


For any $I=\left\{i_{0}, \ldots, i_{k}\right\} \in \Lambda_{k}$ let $e_{I} \in \mathbb{G}(k, n)$ be the point corresponding to $e_{i_{0}} \wedge \cdots \wedge e_{i_{k}} \in \wedge^{k+1} \mathbb{C}^{n+1}$. We will denote by $Z_{I}$ the Plücker coordinates on $\mathbb{P}\left(\bigwedge^{k+1} \mathbb{C}^{n+1}\right)$.

From [MR19] we have a notion of distance in $\Lambda_{k}$ given by

$$
d(I, J)=|I|-|I \cap J|
$$

for all $I, J \in \Lambda_{k}$. More generally, we define

$$
\Lambda=\Lambda_{k_{1}} \times \cdots \times \Lambda_{k_{r}}
$$

Given $I=\left\{I^{1}, \ldots, I^{r}\right\} \in \Lambda$ let $e_{I} \in \prod_{i=1}^{r} \mathbb{G}\left(k_{i}, n\right)$ be the point corresponding to $e_{I^{1}} \otimes \cdots \otimes e_{I^{r}} \in \mathbb{P}^{N}$, and by $Z_{I}$ the corresponding homogeneous coordinate of $\mathbb{P}^{N}$. Furthermore, for all $I, J \in \Lambda$ with $I=\left\{I^{1}, \ldots, I^{r}\right\}$ and $J=\left\{J^{1}, \ldots, J^{r}\right\}$, we define their distance as

$$
d(I, J)=\sum_{i=1}^{r} d\left(I^{i}, J^{i}\right)
$$

where $d\left(I^{i}, J^{i}\right)$ is the distance defined in (2.1).

From now on we will assume that $n \geq 2 k_{r}+1$. Under this assumption $\Lambda$ has diameter $r+\sum_{i=1}^{r} k_{i}$ with respect to this distance.

In the following, we give an explicit description of the osculating spaces of $\prod_{i=1}^{r} \mathbb{G}\left(k_{i}, n\right)$ at coordinate points.

Proposition 2.2. For each $s \geq 0$

$$
T_{e_{I}}^{s}\left(\prod_{i=1}^{r} \mathbb{G}\left(k_{i}, n\right)\right)=\left\langle e_{J} ; d(I, J) \leq s\right\rangle=\left\{Z_{J}=0 ; d(I, J)>s\right\} \subset \mathbb{P}^{N}
$$

In particular, $T_{e_{I}}^{s}\left(\prod_{i=1}^{r} \mathbb{G}\left(k_{i}, n\right)\right)=\mathbb{P}^{N}$ for $s \geq r+\sum_{i=1}^{r} k_{i}$.

Proof. Set $I=\left\{I^{1}, \ldots, I^{r}\right\} \in \Lambda$. We may assume that $I^{i}=\left\{0, \ldots, k_{i}\right\}$ for each $1 \leq i \leq r$ and consider the following parametrization of $\prod_{i=1}^{r} \mathbb{G}\left(k_{i}, n\right)$ in a neighborhood of $e_{I}$ :

$$
\begin{aligned}
& \varphi: \prod_{i=1}^{r} \mathbb{C}^{\left(k_{i}+1\right)\left(n-k_{i}\right)} \longrightarrow \mathbb{P}^{N}
\end{aligned}
$$

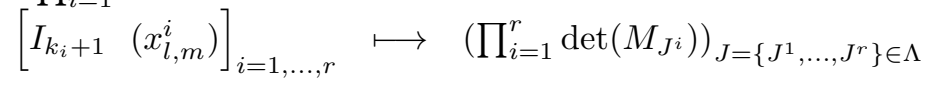

where $M_{J^{i}}$ is the submatrix obtained from $\left[I_{k_{i}+1},\left(x_{l, m}^{i}\right)_{\substack{0 \leq l \leq k_{i} \\ k_{i}+1 \leq m \leq n}}\right]$ by considering the columns indexed by $J^{i}$.

For each $J \in \Lambda$, we will denote $\prod_{i=1}^{r} \operatorname{det}\left(M_{J^{l}}\right)$ simply by $\operatorname{det}\left(M_{J}\right)$. Note that each variable appears in degree at most one in the coordinates of $\varphi$. Therefore, deriving two times with respect to the same variable always gives zero. Furthermore, as $\operatorname{det}\left(M_{J}\right)$ has degree at most $r+\sum_{i=1}^{r} k_{i}$ all partial derivatives of order greater or equal than $r+\sum_{i=1}^{r} k_{i}$ are zero. Thus, it is enough to prove the claim for $s \leq r+\sum_{i=1}^{r} k_{i}$.

Given $J=\left\{J^{1}, \ldots, J^{r}\right\} \in \Lambda$, let $i, k, k^{\prime}$ be integers such that $1 \leq i \leq r, k \in\left\{0 \ldots, k_{i}\right\}$ and $k^{\prime} \in\left\{k_{i}+1, \ldots, n\right\}$. Then

$$
\frac{\partial \operatorname{det}\left(M_{J}\right)}{\partial x_{k, k^{\prime}}^{i}}=\left\{\begin{array}{cl} 
\pm \ldots \operatorname{det}\left(M_{J^{i-1}}\right) \operatorname{det}\left(M_{J^{i}, k, k^{\prime}}\right) \operatorname{det}\left(M_{J^{i+1}}\right) \ldots & k^{\prime} \in J^{i} \\
0 & k^{\prime} \notin J^{i}
\end{array}\right.
$$

where $M_{J^{i}, k, k^{\prime}}$ is the submatrix obtained from $M_{J^{i}}$ by deleting the column indexed by $k^{\prime}$ and the row indexed by $k$.

More generally, let $m_{1}, \ldots, m_{r}$ be non-negative integers such that their sum is bigger than one. For each $i=1, \ldots, r$ consider

$$
K_{i}=\left\{k_{1}^{i}, \ldots, k_{m_{i}}^{i}\right\} \subset\left\{0, \ldots, k_{i}\right\} \text { and } K_{i}^{\prime}=\left\{k_{1}^{\prime i}, \ldots, k_{m_{i}}^{\prime i}\right\} \subset\left\{k_{i}+1, \ldots, n\right\}
$$

with $\left|K_{i}\right|=\left|K_{i}^{\prime}\right|=m_{i}$. Now, set $m=m_{1}+\cdots+m_{r}$ and

$$
K=\left\{K_{1}, \ldots, K_{r}\right\}, K^{\prime}=\left\{K_{1}^{\prime}, \ldots, K_{r}^{\prime}\right\}
$$

Therefore, denoting $\partial x_{k_{1}^{1}, k_{1}^{\prime 1}}^{1} \cdots \partial x_{k_{m_{r}}^{r}, k_{m_{r}}^{\prime r}}^{r}$ simply by $\partial^{m} K, K^{\prime}$ we have

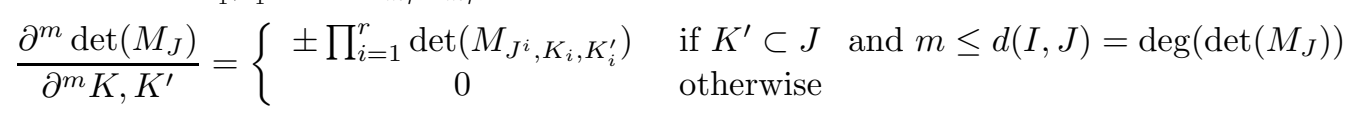

for any $J \in \Lambda$, where $K^{\prime} \subset J$ means that $\left\{k_{1}^{\prime 1}, \ldots, k_{m_{1}}^{\prime 1}\right\} \subset J^{1}, \ldots,\left\{k_{1}^{\prime r}, \ldots, k_{m_{r}}^{\prime r}\right\} \subset J^{r}$, and $M_{J^{i}, K_{i}, K_{i}^{\prime}}$ is the submatrix obtained from $M_{J^{i}}$ deleting the columns indexed by $K_{i}^{\prime}$ and the rows indexed by $K_{i}$. Thus,

$$
\frac{\partial^{m} \operatorname{det}\left(M_{J}\right)}{\partial^{m} K, K^{\prime}}(0)=\left\{\begin{array}{cl} 
\pm 1 & \text { if } J^{i}=K_{i}^{\prime} \cup\left(\left\{I^{i} \backslash K_{i}\right\}\right) \text { for each } i=1, \ldots, r \\
0 & \text { otherwise }
\end{array}\right.
$$


Finally, let us denote by $J=K^{\prime} \cup\{I \backslash K\}$ the element in $\Lambda$ for which $J^{i}=K_{i}^{\prime} \cup\left(\left\{I^{i} \backslash K_{i}\right\}\right)$ for each $i=1, \ldots, r$. Then, we have that

$$
\frac{\partial^{m} \varphi}{\partial^{m} K, K^{\prime}}(0)= \pm e_{K^{\prime} \cup(\{I \backslash K\})}
$$

Note that $d\left(I, K^{\prime} \cup\{I \backslash K\}\right)=m$, and any $J \in \Lambda$ with $d(I, J)=m$ may be written as $K^{\prime} \cup\{I \backslash K\}$. Thus, we get that

$$
\left\langle\frac{\partial^{m} \varphi}{\partial^{m} K, K^{\prime}}(0) \mid m \leq s\right\rangle=\left\langle e_{J} \mid d(I, J) \leq s\right\rangle
$$

which proves the claim.

Now, it is immediate to compute the dimension of the osculating spaces of $\prod_{i=1}^{r} \mathbb{G}\left(k_{i}, n\right)$.

Corollary 2.4. For any point $p \in \prod_{i=1}^{r} \mathbb{G}\left(k_{i}, n\right)$ we have

$$
\operatorname{dim} T_{p}^{s}\left(\prod_{i=1}^{r} \mathbb{G}\left(k_{i}, n\right)\right)=\sum_{\substack{i=1, \ldots, r \\
0 \leq s_{i} \leq k_{i}+1, s_{1}+\cdots+s_{r} \leq s}}\left(\begin{array}{c}
n-k_{1} \\
s_{1}
\end{array}\right)\left(\begin{array}{c}
k_{1}+1 \\
s_{1}
\end{array}\right) \cdots\left(\begin{array}{c}
n-k_{r} \\
s_{r}
\end{array}\right)\left(\begin{array}{c}
k_{r}+1 \\
s_{r}
\end{array}\right)
$$

for any $0 \leq s<r+\sum_{i=1}^{r} k_{i}$ while $T_{p}^{s}\left(\prod_{i=1}^{r} \mathbb{G}\left(k_{i}, n\right)\right)=\mathbb{P}^{N}$ for any $s \geq r+\sum_{i=1}^{r} k_{i}$.

Proof. Since the general linear group $G L(n+1)$ acts transitively on $\prod_{i=1}^{r} \mathbb{G}\left(k_{i}, n\right)$ the statement follows from Proposition 2.2

2.4. Osculating Projections. For a general point $p \in \prod_{i=1}^{r} \mathbb{G}\left(k_{i}, n\right)$, we will denote $T_{p}^{s}\left(\prod_{i=1}^{r} \mathbb{G}\left(k_{i}, n\right)\right)$ simply by $T_{p}^{s}$. Now, take $0 \leq s \leq r+\sum_{i=1}^{r} k_{i}$ and $I \in \Lambda$. By Proposition 2.2 the linear projection of $\prod_{i=1}^{r} \mathbb{G}\left(k_{i}, n\right)$ from $T_{e_{I}}^{s}$ is given by

$$
\begin{aligned}
& \Pi_{T_{e_{I}}^{s}}: \prod_{i=1}^{r} \mathbb{G}\left(k_{i}\right) \quad \rightarrow \mathbb{P}^{N_{s}^{\prime}} \\
& \left(Z_{J}\right)_{J \in \Lambda} \longmapsto\left(Z_{J}\right)_{J \in \Lambda \mid d(I, J)>s}
\end{aligned}
$$

Moreover, given $I^{\prime} \subset\{0, \ldots, n\}$ with $\left|I^{\prime}\right|=m$ we have the linear projection

$$
\begin{aligned}
\pi_{I^{\prime}}: \mathbb{P}^{n} & \rightarrow \mathbb{P}^{n-m} \\
& \left(x_{i}\right) \stackrel{\longmapsto}{ }\left(x_{i}\right)_{i \in\{0, \ldots, n\} \backslash I^{\prime}}
\end{aligned}
$$

which in turns induces the linear projection

$$
\begin{aligned}
& \Pi_{I^{\prime}}: \quad \mathbb{G}(k, n) \quad \rightarrow \quad \mathbb{G}(k, n-m) \\
& V \quad \longmapsto\left\langle\pi_{I^{\prime}}(V)\right\rangle \\
& \left(Z_{J}\right)_{J \in \Lambda_{k}} \longmapsto\left(Z_{J}\right)_{J \in \Lambda_{k} \mid J \cap I^{\prime}=\emptyset}
\end{aligned}
$$

whenever $k<n-m$.

Finally, let us fix $I=\left\{I^{1}, \ldots, I^{r}\right\} \in \Lambda$ and take $m_{1}, \ldots, m_{r}$ integers such that $m_{i} \leq k_{i}+1$ for each $i=1, \ldots, r$. Then, given $I^{\prime 1} \subset I^{1}, \ldots, I^{\prime r} \subset I^{r}$, with $\left|I^{\prime i}\right|=m_{i}$, we have a projection

$$
\begin{aligned}
& \prod_{i=1}^{r} \Pi_{I^{\prime i}}: \prod_{i=1}^{r} \mathbb{G}\left(k_{i}, n\right) \rightarrow \prod_{i=1}^{r} \mathbb{G}\left(k_{i}, n-m_{i}\right) \\
& V_{1} \times \cdots \times V_{r} \longmapsto \Pi_{I^{\prime 1}}\left(V_{1}\right) \times \cdots \times \Pi_{I^{\prime r}}\left(V_{r}\right)
\end{aligned}
$$

Note that a general fiber of $\prod_{i=1}^{r} \Pi_{I^{\prime} i}$ is isomorphic to $\prod_{i=1}^{r} \mathbb{G}\left(k_{i}, k_{i}+m_{i}\right)$. Indeed, let $x=\prod_{i=1}^{r} \Pi_{I^{\prime i}}\left(\left(V_{i}\right)_{i=1}^{r}\right) \in$ $\prod_{i=1}^{r} \mathbb{G}\left(k_{i}, n-m_{l}\right)$ be a general point. Then, we have

$$
\overline{\left(\prod_{i=1}^{r} \Pi_{I^{\prime i}}\right)^{-1}(x)}=\left\{\left(W_{i}\right)_{i=1}^{r} \in \prod_{i=1}^{r} \mathbb{G}\left(k_{i}, n\right) \mid W_{i} \subset\left\langle V_{i}, e_{j_{1}^{i}}, \ldots, e_{j_{m_{i}}^{i}}\right\rangle, i=1, \ldots, r\right\} .
$$

Lemma 2.5. Let us fix $I=\left\{I^{1}, \ldots, I^{r}\right\} \in \Lambda$. If $0 \leq s \leq r-2+\sum_{i=1}^{r} k_{i}$ and $I^{\prime i} \subset I^{i}$ with $\left|I^{\prime i}\right|=m_{i}$ for each $i=1, \ldots, r$, then the rational map $\Pi_{T_{e_{I}}^{s}}$ factors through $\prod_{i=1}^{r} \Pi_{I^{\prime i}}$ whenever $\sum_{i=1}^{r} m_{i}=s+1$.

Proof. Since the diameter of $\Lambda$ is $r+\sum k_{i}$ we have $\{J \in \Lambda \mid d(I, J) \leq s\} \subsetneq \Lambda$ and then $\Pi_{T_{e_{I}}^{s}}$ is well-defined.

On the other hand, if $J=\left\{J^{1}, \ldots, J^{r}\right\} \in \Lambda$ is such that $J^{i} \cap I^{\prime i}=\emptyset$ for all $i=1, \ldots, r$, then $d(I, J) \geq$ $\sum_{i=1}^{r} m_{i}>s$ which yields that the center of $\Pi_{T_{e_{I}}^{s}}$ is contained in the center of $\prod_{i=1}^{r} \Pi_{I^{\prime i}}$.

Proposition 2.6. The rational map $\Pi_{T_{e_{I}}^{s}}$ is birational for all $0 \leq s \leq r-2+\sum_{i=1}^{r} k_{i}$. 
Proof. Since $T_{e_{I}}^{s}$ contains $T_{e_{I}}^{s-1}$ it is enough to prove the statement for $s=r-2+\sum_{i=1}^{r} k_{i}$. Let us fix $m \in\{1, \ldots, r\}$. By Lemma 2.5, for each subset $I^{\prime m} \subset I^{m}$ with $\left|I^{\prime m}\right|=k_{m}$ there is a rational map $\pi_{I^{\prime m}}$ that makes the following diagram commutative.

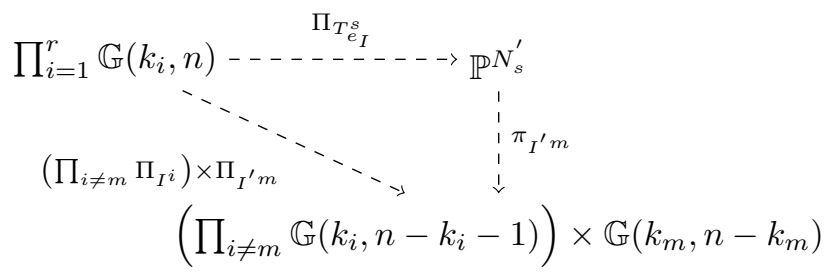

Let $x=\Pi_{T_{e_{I}}^{s}}\left(\left\{V_{i}\right\}_{i=1}^{r}\right)$ be a general point and $X \subset \prod_{i=1}^{r} \mathbb{G}\left(k_{i}, n\right)$ be the fiber of $\Pi_{T_{e_{I}}^{s}}$ over $x$. Set $x_{I^{\prime m}}=\pi_{I^{\prime m}}(x)$, and denote by $X_{I^{\prime m}} \subset \prod_{i=1}^{r} \mathbb{G}\left(k_{i}, n\right)$ the fiber of $\left(\prod_{i \neq m} \Pi_{I^{i}}\right) \times \Pi_{I^{\prime m}}$ over $x_{I^{\prime m}}$. Thus,

$$
X \subset \bigcap_{I^{\prime m}} X_{I^{\prime m}}
$$

where this intersection runs over all $I^{\prime m} \subset I^{m}$ with $\left|I^{\prime m}\right|=k_{m}$ and $m=1, \ldots, r$. Now, if $\left(W_{i}\right)_{i=1}^{r}$ is a general point in $X$ then

$$
W_{m} \subset\left\langle e_{j_{1}}, \ldots, e_{j_{k_{m}}}, V_{m}\right\rangle \text { for any } I^{\prime m}=\left\{e_{j_{1}}, \ldots, e_{j_{k_{m}}}\right\} \subset I^{m}
$$

Therefore,

$$
W_{m} \subset \bigcap_{I^{\prime m}}\left\langle e_{j_{1}}, \ldots, e_{j_{k}}, V_{m}\right\rangle=V_{m}
$$

This implies $W_{m}=V_{m}$ for every $m=1, \ldots, r$. Since we are working in characteristic zero, we conclude that $\Pi_{T_{e_{I}}^{s}}$ is birational.

The next step is to study linear projections from the span of several osculating spaces. In particular, we want to understand when such a projection is birational. First of all, note that the order of osculating spaces can not exceed $r-2+\sum_{i=1}^{r} k_{i}$. Furthermore, in order to carry out the computations, we need to consider just the coordinates points of $\prod_{i=1}^{r} \mathbb{G}\left(k_{i}, n\right)$ such that the corresponding linear subspaces are linearly independent in $\mathbb{C}^{n+1}$, then we can use at most

$$
\alpha:=\left\lfloor\frac{n+1}{k_{r}+1}\right\rfloor
$$

of them. Now, let us consider the points $e_{I_{1}}, \ldots, e_{I_{\alpha}} \in \prod_{i=1}^{r} \mathbb{G}\left(k_{i}, n\right)$, where

$$
\begin{aligned}
& I_{1}=\left\{I_{1}^{1}=\left\{0, \ldots, k_{1}\right\}, \ldots, I_{1}^{r}=\left\{0, \ldots, k_{r}\right\}\right\} \\
& I_{2}=\left\{I_{2}^{1}=\left\{k_{r}+1, \ldots, k_{r}+k_{1}+1\right\}, \ldots, I_{2}^{r}=\left\{k_{r}+1, \ldots, k_{r}+k_{r}+1\right\}\right\} \\
& \vdots \\
& I_{\alpha}=\left\{\ldots, I_{\alpha}^{i}=\left\{\left(k_{r}+1\right)(\alpha-1), \ldots,\left(k_{r}+1\right)(\alpha-1)+k_{i}\right\}, \ldots\right\}
\end{aligned}
$$

Let $s_{1}, \ldots, s_{\alpha}$ be integers such that $0 \leq s_{m} \leq r-2+\sum_{i=1}^{r} k_{i}$. Denote the linear subspace $\left\langle T_{e_{I_{1}}}^{s_{1}}, \ldots, T_{e_{I_{m}}}^{s_{m}}\right\rangle$ simply by $T_{e_{1}, \ldots, e_{I_{m}}}^{s_{1}, \ldots, s_{m}}$. Then, for $m \leq \alpha$ we have the linear projection

$$
\begin{aligned}
\prod_{T_{e_{1}, \ldots, e_{I_{m}}}^{s_{1}, \ldots, s_{m}}}: \prod_{i=1}^{r} \mathbb{G}\left(k_{i}, n\right) & \rightarrow \mathbb{P}^{N_{s_{1}, \ldots, s_{m}}^{\prime}} \\
\left(Z_{J}\right)_{J \in \Lambda} & \longmapsto\left(Z_{J}\right)_{J \in \Lambda \mid d\left(J, I_{1}\right)>s_{1}, \ldots, d\left(J, I_{m}\right)>s_{m}}
\end{aligned}
$$

Now, consider $I_{1}, \ldots, I_{\alpha}$ as in (2.7), and $I_{m}^{\prime i} \subset I_{m}^{i}$ with $\left|I_{m}^{\prime i}\right|=s_{m}^{i}$ for each $1 \leq m \leq \alpha$ and $i=1, \ldots, r$, where $s_{m}^{i}$ are non-negative integers. If $I^{\prime i}$ denotes the union $\bigcup_{m=1}^{\alpha} I_{m}^{\prime i}$, then for each $i=1, \ldots, r$ we have a linear projection of $\mathbb{P}^{n}$

$$
\begin{array}{llll}
\pi_{I^{\prime i}}: & \mathbb{P}^{n} & -\rightarrow & \mathbb{P}^{n-\sum_{m=1}^{\alpha} s_{m}^{i}} \\
& \left(x_{i}\right)_{0 \leq i \leq n} & \longmapsto & \left(x_{i}\right)_{0 \leq i \leq n} \text { and } i \notin I^{\prime i}
\end{array}
$$

which in turns induces the following projection

$$
\begin{aligned}
\Pi_{I^{\prime i}}: \mathbb{G}\left(k_{i}, n\right) & -\rightarrow \mathbb{G}\left(k_{i}, n-\sum_{m=1}^{\alpha} s_{m}^{i}\right) \\
V & \longmapsto\left\langle\pi_{I^{\prime i}}(V)\right\rangle \\
\left(Z_{J}\right)_{J \in \Lambda_{k_{i}}} & \longmapsto\left(Z_{J}\right)_{J \in \Lambda_{k_{i}} \mid J \cap I^{\prime i}=\emptyset}
\end{aligned}
$$


whenever $n-\sum_{m=1}^{\alpha} s_{m}^{i} \geq k_{i}$. Finally, if $n-\sum_{m=1}^{\alpha} s_{m}^{i} \geq k_{i}$ for each $i=1, \ldots, r$, then the projections above induce a projection

$$
\begin{aligned}
\prod_{i=1}^{r} \Pi_{I^{\prime i}}: \quad \prod_{i=1}^{r} \mathbb{G}\left(k_{i}, n\right) & \rightarrow \prod_{i=1}^{r} \mathbb{G}\left(k_{i}, n-\sum_{m=1}^{\alpha} s_{m}^{i}\right) \\
\left(V_{1}, \ldots, V_{r}\right) & \longmapsto\left(\prod_{I^{\prime}}\left(V_{1}\right), \ldots, \Pi_{I^{\prime r}}\left(V_{r}\right)\right)
\end{aligned}
$$

Lemma 2.8. Let $I_{1}, \ldots, I_{\alpha}$ be as in 2.7), $m, s_{1}, \ldots, s_{m}$ integers such that $1<m \leq \alpha$ and $0 \leq s_{i} \leq r-$ $2+\sum_{i=1}^{r} k_{i}$. Now, consider $I_{1}^{\prime i} \subset I_{1}^{i}, \ldots, I_{m}^{\prime i} \subset I_{m}^{i}$ with $\left|I_{j}^{\prime i}\right|=s_{j}^{i}$, where $s_{j}^{i}$ is a non-negative integer for each $i=1, \ldots, r$ and $1 \leq j \leq m$. For $j>m$ and $i=1, \ldots, r$ set $I_{j}^{\prime i}=\emptyset \subset I_{j}^{i}$. Denote by $I^{\prime i}$ the union $\bigcup_{j=1}^{\alpha} I_{j}^{\prime i}$ for each $i=1, \ldots, r$ and assume that

(i) $n-\sum_{j=1}^{m} s_{j}^{i} \geq k_{i}$ for each $i=1, \ldots, r$;

(ii) $\sum_{i=1}^{r} s_{j}^{i} \geq s_{j}+1$ for each $j=1, \ldots, m$.

Then, the rational maps $\prod_{i=1}^{r} \prod_{I^{\prime i}}$ and $\prod_{T_{I_{I_{1}}, \ldots, e_{I_{m}}}^{s_{1}, \ldots, s_{m}}}$ are well-defined and the former factors through the latter.

Proof. Note that $\Pi_{T_{e_{I_{1}}, \ldots, e_{I}}^{s_{1}, \ldots, s_{m}}}$ is well-defined if and only if $\left\{J \in \Lambda \mid d\left(J, I_{1}\right)>s_{1}, \ldots, d\left(J, I_{m}\right)>s_{m}\right\} \neq \emptyset$. From (i) we have that for each $1 \leq i \leq r$ the set $\{0, \ldots, n\} \backslash I^{i}$ has at least $k_{i}+1$ elements. Therefore, we have a set $J^{i} \subset\{0, \ldots, n\} \backslash I^{\prime i}$ of cardinality $k_{i}+1$ and taking $J=\left\{J^{1}, \ldots, J^{r}\right\} \in \Lambda$ we have

$$
d\left(I_{j}, J\right)=\sum_{i=1}^{r} d\left(I_{j}^{i}, J^{i}\right) \geq \sum_{i=1}^{r} s_{j}^{i}=s_{j}+1>s_{j}
$$

for each $1 \leq j \leq m$. Hence, $\prod_{T_{e_{1}, \ldots, \ldots, I_{m}}^{s_{1}, \ldots, s_{m}}}$ is well-defined. Now, note that (i) yields that $\prod_{i=1}^{r} \prod_{I^{\prime i}}$ is well-defined. Furthermore, if $J \in \Lambda$ and $J^{i} \cap I^{\prime i}=\emptyset$ for all $i=1, \ldots, r$, then $d\left(J, I_{1}\right)>s_{1}, \ldots, d\left(J, I_{m}\right)>s_{m}$. Thus, the center of $\prod_{T_{e_{1}, \ldots, e_{I_{m}}}^{s_{1}, \ldots, s_{m}}}$ is contained in the center of $\prod_{i=1}^{r} \Pi_{I^{\prime i}}$.

Proposition 2.9. Let $I_{1}, \ldots, I_{\alpha-1}$ be as in 2.7) and $s_{1}, \ldots, s_{\alpha-1}$ be integers such that $0 \leq s_{j} \leq s=r-2+$ $\sum_{i=1}^{r} k_{i}$. Then, the projection $\Pi_{T_{I_{1}}, \ldots, e_{I_{\alpha-1}}}^{s_{1}, \ldots, s_{\alpha-1}}$ is birational.

Proof. Fix $m \in\{1, \ldots, r\}$. For any $j=1, \ldots, \alpha-1$ consider $I_{j}^{\prime m} \subset I_{j}^{m}$ with $\left|I_{j}^{\prime m}\right|=k_{m}$ and $I_{j}^{\prime i}=I_{j}^{i}$ for $i \neq m$. Set $I^{\prime i}=\bigcup_{j=1}^{\alpha-1} I_{j}^{\prime i}$, then

$$
n-(\alpha-1)\left(k_{i}+1\right) \geq n-(\alpha-1)\left(k_{r}+1\right) \geq n-\frac{\left(n-k_{r}\right)}{k_{r}+1}\left(k_{r}+1\right) \geq k_{r} \geq k_{i}
$$

and

$$
n-(\alpha-1) k_{m} \geq n-\frac{\left(n-k_{r}\right)}{k_{r}+1} k_{r}=\frac{n k_{r}+n-n k_{r}+k_{r}^{2}}{k_{r}+1} \geq \frac{2 k_{r}+1+k_{r}^{2}}{k_{r}+1} \geq k_{m}+1
$$

Thus, our set of subsets $I_{j}^{\prime i}$ satisfies (i) in Lemma 2.8 Furthermore, for each $j=1, \ldots, \alpha-1$

$$
\sum_{i=1}^{r}\left|I_{j}^{\prime l}\right|=k_{m}+\sum_{i \neq m}\left(k_{i}+1\right)=r-1+\sum_{i=1}^{r} k_{i}=s+1
$$

Therefore, by Lemma 2.8 there exists a rational map $\pi_{I^{\prime m}}$ that makes the following diagram commutative

$$
\begin{aligned}
& \prod_{i=1}^{r} \mathbb{G}\left(k_{i}, n\right) \stackrel{\prod_{T_{e_{I}}, \ldots, e_{I_{\alpha-1}}}}{\cdots} \mathbb{N}^{N_{s, \ldots, s}^{\prime}}
\end{aligned}
$$

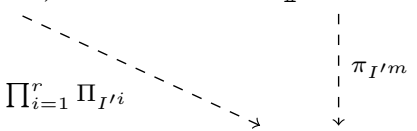

$$
\begin{aligned}
& \prod_{i=1}^{r} \mathbb{G}\left(k_{i}, n-\sum_{j=1}^{\alpha-1}\left|I_{j}^{\prime i}\right|\right)
\end{aligned}
$$

Now, let $x=\prod_{T_{e_{1}}^{s, \ldots ., e_{I_{\alpha-1}}}}\left(\left\{V_{i}\right\}_{i=1}^{r}\right)$ be a general point in the image of $\Pi_{T_{e_{1}}, \ldots, e_{I_{\alpha-1}}, \ldots, s}$, and $X \subset \prod_{i=1}^{r} \mathbb{G}\left(k_{i}, n\right)$ be the fiber of $\prod_{T_{e_{1}, \ldots, e_{I_{\alpha-1}}}^{s, \ldots, s}}$ over $x$. Set $x_{I^{\prime m}}=\pi_{I^{\prime m}}(x)$ and denote by $X_{I^{\prime m}} \subset \prod_{i=1}^{r} \mathbb{G}\left(k_{i}, n\right)$ the fiber of $\prod_{i=1}^{r} \Pi_{I^{\prime i}}$ over $x_{I^{\prime m}}$. Therefore, $X \subset \bigcap_{I^{\prime m}} X_{I^{\prime m}}$ where this intersection runs over all subsets $I^{\prime m}=\bigcup_{j=1}^{\alpha-1} I_{j}^{\prime m}$ with $I_{j}^{\prime m} \subset I_{j}^{m}$ and $\left|I_{j}^{\prime m}\right|=k_{m}$. In particular, if $\left\{W_{i}\right\}_{i=1}^{r} \in X$ is a general point, then we must have $W_{m} \subset\left\langle e_{i} \mid i \in I^{\prime m} ; V_{m}\right\rangle$ 
and hence $W_{m} \subset \bigcap_{I^{\prime m}}\left\langle e_{i} \mid i \in I^{\prime m} ; V_{m}\right\rangle$. Now, since $\left|I_{j}^{\prime m}\right|=k_{m}$ we have $\bigcap_{I^{\prime m}}\left\langle e_{i} \mid i \in I^{\prime m}\right\rangle=\emptyset$ and then $V_{m}=\bigcap_{I^{\prime m}}\left\langle e_{i} \mid i \in I^{\prime m} ; V_{m}\right\rangle$ which in turn yields $W_{m}=V_{m}$, for all $m=1, \ldots, r$.

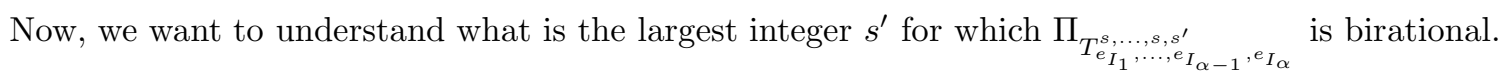

Proposition 2.10. Let $I_{1}, \ldots, I_{\alpha}$ be as in (2.7) and $s=r-2+\sum_{i=1}^{r} k_{i}$. Consider $s_{i}^{\prime}=\min \left\{k_{i}+1, n-\alpha\left(k_{i}+1\right)\right\}$ for $i \neq r, s_{r}^{\prime}=\min \left\{k_{r}, n-\alpha k_{r}-1\right\}$, and set $s^{\prime}=\sum_{i=1}^{r} s_{i}^{\prime}-1 \leq s$. Then,

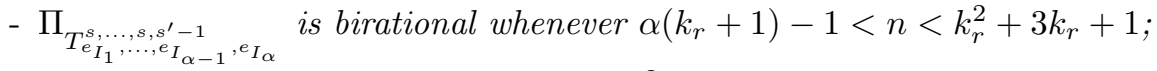

- $\Pi_{T_{e_{I_{1}}, \ldots, e_{I_{\alpha}}}^{s, \ldots, s}}$ is birational whenever $n \geq k_{r}^{2}+3 k_{r}+1$.

Proof. First, let us assume that $s_{r}^{\prime}<k_{r}$, that is $n-\alpha k_{r}-1<k_{r}$, or equivalently

$$
n-\alpha k_{r}<k_{r}+1 \Leftrightarrow n-\frac{(n+1)}{k_{r}+1} k_{r}<k_{r}+1 \Leftrightarrow n<k_{r}^{2}+3 k_{r}+1
$$

Now, fix a pair of indexes $(l, m) \in\{1, \ldots, \alpha-1\} \times\{1, \ldots, r\}$ and consider subsets $I_{j}^{\prime i} \subseteq I_{j}^{i}$ with $\left|I_{j}^{\prime i}\right|=a_{j, i}$ for each $i \in\{1, \ldots, r\}$ and $j \in\{1, \ldots \alpha\}$ such that

$$
a_{j, i}= \begin{cases}k_{i} & \text { if } \quad i=m, j=l \text { or } i=r, j \neq l, \alpha ; \\ k_{i}+1 & \text { if } i=r \neq m, j=l \text { or } i \neq m, r \text { or } l \neq m, \alpha ; \\ s_{i}^{\prime} & \text { if } j=\alpha, i \neq m ; \\ s_{m}^{\prime}-1 & \text { if } j=\alpha, i=m .\end{cases}
$$

Note that, since $\alpha\left(k_{r}+1\right)-1<n$ we have $a_{j, i} \geq 0$ for all $j \in\{1, \ldots, \alpha\}$ and $i \in\{1, \ldots, r\}$. Moreover, if $m \neq r$ then

$$
n-\sum_{j=1}^{\alpha}\left|I_{j}^{\prime m}\right|=n-(\alpha-2)\left(k_{m}+1\right)-k_{m}-\left|I_{\alpha}^{\prime m}\right| \geq n-(\alpha-1)\left(k_{m}+1\right)-\left(n-\alpha\left(k_{m}+1\right)-1\right)=k_{m}+2
$$

and $n-\sum_{j=1}^{\alpha}\left|I_{j}^{\prime r}\right|=n-(\alpha-2) k_{r}-\left(k_{r}+1\right)-\left|I_{\alpha}^{\prime r}\right| \geq n-(\alpha-1) k_{r}-1-\left(n-\alpha k_{r}-1\right)=k_{r}$. If $r=m$ we have

$$
n-\sum_{j=1}^{\alpha}\left|I_{j}^{\prime r}\right|=n-(\alpha-2) k_{r}-\left(k_{r}+1\right)-\left|I_{\alpha}^{\prime r}\right| \geq n-(\alpha-1) k_{r}-1-\left(n-\alpha k_{r}-2\right)=k_{r}+1
$$

Finally, for $i \neq m, r$ we have

$$
n-\sum_{j=1}^{\alpha}\left|I_{j}^{\prime i}\right|=n-(\alpha-1)\left(k_{i}+1\right)-\left|I_{\alpha}^{\prime i}\right| \geq n-(\alpha-1)\left(k_{i}+1\right)-\left(n-\alpha\left(k_{i}+1\right)\right)=k_{i}+1
$$

This yields that (i) in Lemma 2.8 is satisfied by the sets $I_{j}^{\prime i}$. Moreover, (ii) is satisfied as well. Then, by Lemma 2.8 there exists a rational map $\pi_{I_{l}^{\prime m}, I_{\alpha}^{\prime m}}$ making the following diagram commutative

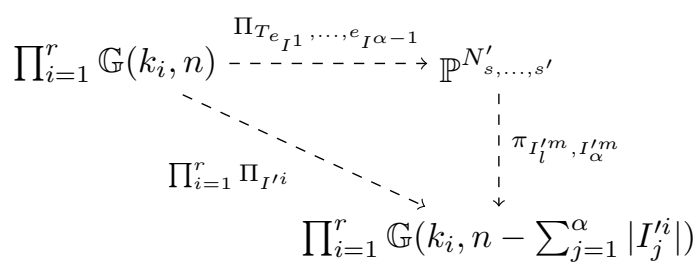

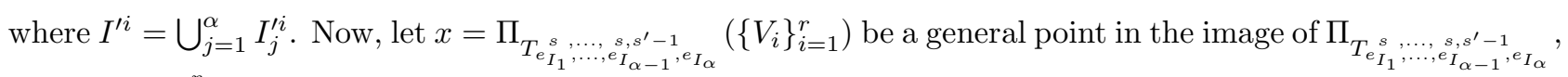

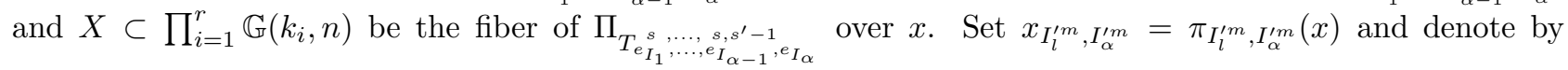
$X_{I_{l}^{\prime m}, I_{\alpha}^{\prime m}} \subset \prod_{i=1}^{r} \mathbb{G}\left(k_{i}, n\right)$ the fiber of $\prod_{i=1}^{r} \Pi_{I^{\prime i}}$ over $x_{I_{l}^{\prime m}, I_{\alpha}^{\prime m}}$. Therefore, $X \subset \bigcap_{I_{l}^{\prime m}, I_{\alpha}^{\prime m}} X_{I_{l}^{\prime m}, I_{\alpha}^{\prime m}}$, where the intersection runs over all pairs of sets $I_{l}^{\prime m}$ and $I_{\alpha}^{\prime m}$ with $\left|I_{l}^{\prime m}\right|=k_{m}$ and $\left|I_{\alpha}^{\prime m}\right|=s_{m}^{\prime}-1$, and for all pairs of indexes $(l, m) \in\{1, \ldots, r\} \times\{1, \ldots, \alpha-1\}$. In particular, if $\left\{W_{i}\right\}_{i=1}^{r} \in X$ is a general point then for every $m \in\{1, \ldots, r\}$ we have $W_{m} \subset \bigcap_{I_{l}^{\prime m}, I_{\alpha}^{\prime m}}\left\langle e_{i} \mid i \in I^{\prime m} ; V_{m}\right\rangle$, where the intersection runs over all pair of sets $I_{l}^{\prime m}$ and 
$I_{\alpha}^{\prime m}$ with $\left|I_{l}^{\prime m}\right|=k_{m}$ and $\left|I_{\alpha}^{\prime m}\right|=s_{m}^{\prime}-1$, and $l \in\{1, \ldots, \alpha-1\}$. Now, since $\left|I_{l}^{\prime m}\right|=k_{m}, s_{m}^{\prime}-1 \leq k_{m}$ and $l \in\{1, \ldots, \alpha-1\}$, we must have $\bigcap_{I_{l}^{\prime m}, I_{\alpha}^{\prime m}}\left\langle e_{i} \mid i \in I^{\prime m}\right\rangle=\emptyset$ and then $V_{m}=\bigcap_{I^{\prime m}}\left\langle e_{i} \mid i \in I^{\prime m} ; V_{m}\right\rangle$ which yields $W_{m}=V_{m}$, for all $m=1, \ldots, r$.

Now, assume that $n \geq k_{r}^{2}+k_{r}+1$. In this case we have that

$$
n-\alpha k_{r}-1 \geq n-\frac{(n+1)}{k_{r}+1} k_{r}-1=\frac{n\left(k_{r}+1\right)-(n+1) k_{r}-\left(k_{r}+1\right)}{k_{r}+1} \geq k_{r}
$$

and for $i<r$ we have

$$
n-\alpha\left(k_{i}+1\right) \geq n-\alpha\left(k_{r}\right) \geq n-\frac{(n+1)}{k_{r}+1} k_{r}=\frac{n-k_{r}}{k_{r}+1} \geq \frac{k_{r}^{2}+3 k_{r}+1-k_{r}}{k_{r}+1}=k_{r}+1>k_{i}+1
$$

Now, for each pair of indexes $(l, m) \in\{1, \ldots, \alpha\} \times\{1, \ldots, r\}$ we can consider subsets $I_{j}^{\prime i} \subseteq I_{j}^{i}$ with $\left|I_{j}^{\prime i}\right|=a_{j, i}$ for each $i \in\{1, \ldots, r\}$ and $j \in\{1, \ldots \alpha\}$ such that

$$
a_{j, i}= \begin{cases}k_{i} & \text { if } \quad i=m, j=l \text { or } i=r, j \neq l \\ k_{i}+1 & \text { if } i=r \neq m, j=l \text { or } i \neq m, r \text { or } l \neq m .\end{cases}
$$

Therefore, arguing as in the proof of the first claim we conclude that $\prod_{T_{e_{I_{1}}, \ldots, e_{I_{\alpha}}}^{s, \ldots, s}}$ is birational.

2.10. Degenerating tangential projections to osculating projections. In this section we study how the notion of osculating regularity introduced in MR19] behaves for products of Grassmannians. Let us recall [MR19, Definition 5.5, Assumption 5.2] and [AMR19, Definition 4.4].

Definition 2.11. Let $X \subset \mathbb{P}^{N}$ be a projective variety. We say that $X$ has $m$-osculating regularity if the following property holds: given general points $p_{1}, \ldots, p_{m} \in X$ and an integer $s \geq 0$, there exists a smooth curve $C$ and morphisms $\gamma_{j}: C \rightarrow X, j=2, \ldots, m$, such that $\gamma_{j}\left(t_{0}\right)=p_{1}, \gamma_{j}\left(t_{\infty}\right)=p_{j}$, and the flat limit $T_{0}$ in the Grassmannian of the family of linear spaces

$$
T_{t}=\left\langle T_{p_{1}}^{s}, T_{\gamma_{2}(t)}^{s}, \ldots, T_{\gamma_{m}(t)}^{s}\right\rangle, t \in C \backslash\left\{t_{0}\right\}
$$

is contained in $T_{p_{1}}^{2 s+1}$. We say that $\gamma_{2}, \ldots, \gamma_{m}$ realize the $m$-osculating regularity of $X$ for $p_{1}, \ldots, p_{m}$.

We say that $X$ has strong 2 -osculating regularity if the following property holds: given general points $p, q \in X$ and integers $s_{1}, s_{2} \geq 0$, there exists a smooth curve $\gamma: C \rightarrow X$ such that $\gamma\left(t_{0}\right)=p, \gamma\left(t_{\infty}\right)=q$ and the flat limit $T_{0}$ in the Grassmannian of the family of linear spaces

$$
T_{t}=\left\langle T_{p}^{s_{1}}, T_{\gamma(t)}^{s_{2}}\right\rangle, t \in C \backslash\left\{t_{0}\right\}
$$

is contained in $T_{p}^{s_{1}+s_{2}+1}$.

For a discussion on the notions of $m$-osculating regularity and strong 2-osculating regularity we refer to [MR19, Section 5] and [AMR19, Section 4].

Proposition 2.12. The variety $\prod_{i=1}^{r} \mathbb{G}\left(k_{i}, n\right)$ has strong 2-osculating regularity.

Proof. Let $p, q \in \prod_{i=1}^{r} \mathbb{G}\left(k_{i}, n\right)$ be general points. We may assume that $p=e_{I_{1}}$ and $q=e_{I_{2}}$ with $I_{1}, I_{2}$ as in (2.7) and consider the degree $r+\sum_{i=1}^{r} k_{i}$ rational normal curve given by

$$
\gamma(s: t)=\prod_{i=1}^{r}\left(s e_{0}+t e_{k_{r}+1}\right) \wedge \cdots \wedge\left(s e_{k_{i}}+t e_{k_{r}+k_{i}+1}\right)
$$

We work on the affine chart $s=1$ and set $t=(1: t) \in \mathbb{P}^{1}$. Now, consider the points

$$
e_{0}, \ldots, e_{n}, e_{0}^{t}=e_{0}+t e_{k_{r}+1}, \ldots, e_{k_{r}}^{t}=e_{k_{r}}+t e_{2 k_{r}+1}, e_{k_{r}+1}^{t}=e_{k_{r}+1}, \ldots, e_{n}^{t}=e_{n}
$$

and, for each $I=\left\{I^{1}, \ldots, I^{r}\right\} \in \Lambda$, the corresponding points in $e_{I}^{t}=e_{I^{1}}^{t} \otimes e_{I^{2}}^{t} \otimes \cdots \otimes e_{I^{r}}^{t} \in \prod_{i=1}^{r} \mathbb{G}\left(k_{i}, n\right)$ where, setting $I^{j}=\left\{i_{1}^{j}, \ldots, i_{k_{j}}^{j}\right\}, e_{I^{j}}^{t}=e_{i_{1}^{j}}^{t} \wedge \cdots \wedge e_{i_{k_{j}}^{j}}^{t}$.

Given integers $s_{1}, s_{2} \geq 0$, let us consider the family of linear spaces

$$
T_{t}=\left\langle T_{e_{I_{1}}}^{s_{1}}, T_{e_{\gamma(t)}}^{s_{2}}\right\rangle, t \in \mathbb{P}^{1} \backslash\{0\}
$$

By Proposition 2.2 we have

$$
T_{t}=\left\langle e_{J}\left|d\left(I_{1}, J\right) \leq s_{1} ; e_{J}^{t}\right| d\left(I_{2}, J\right) \leq s_{2}\right\rangle, t \neq 0
$$


and

$$
T_{e_{1}}^{s_{1}+s_{2}+1}=\left\langle e_{J} \mid d\left(I_{1}, J\right) \leq s_{1}+s_{2}+1\right\rangle=\left\{Z_{J}=0 \mid d\left(I_{1}, J\right)>s_{1}+s_{2}+1\right\}
$$

Now, let $T_{0}$ be the flat limit of $\left\{T_{t}\right\}_{t \in \mathbb{P}^{1} \backslash\{0\}}$, we want to show that $T_{0} \subset T_{p}^{s_{1}+s_{2}+1}$. In order to do this it is enough to exhibit, for each index $I \in \Lambda$ with $d\left(I_{1}, I\right)>s_{1}+s_{2}+1$, a hyperplane $H_{I}$ of type $Z_{I}+t\left(\sum_{J \neq I} f_{J}(t) Z_{J}\right)=0$ where $f_{J}(t) \in \mathbb{C}[t]$ for every $J$. We define, for each $l \geq 0$ and $I=\left\{I^{1}, \ldots, I^{r}\right\} \in \Lambda$,

$$
\Delta(I, l)=\left\{\left\{\left(I^{j} \backslash J^{j}\right) \cup\left(J^{j}+k_{r}+1\right)\right\}_{1 \leq j \leq r} \mid J^{j} \subset I^{j} \cap I_{1}^{j} \text { and } \sum\left|J^{j}\right|=l\right\} \subset \Lambda
$$

Furthermore, for each $l>0$ we define

$$
\begin{gathered}
\Delta(I,-l)=\{J \mid I \in \Delta(J, l)\} ; \\
s_{I}^{+}=\max _{l \geq 0}\{\Delta(I, l) \neq \emptyset\} \in\left\{0, \ldots, \sum k_{j}+r\right\} \\
s_{I}^{-}=\max _{l>0}\{\Delta(I,-l) \neq \emptyset\} \in\left\{0, \ldots, \sum k_{j}+r\right\} \\
\Delta(I)^{+}=\bigcup_{0 \leq l} \Delta(I, l)=\bigcup_{0 \leq l \leq s_{I}^{+}} \Delta(I, l) \\
\Delta(I)^{-}=\bigcup_{0 \leq l} \Delta(I,-l)=\bigcup_{0 \leq l \leq s_{I}^{-}} \Delta(I,-l)
\end{gathered}
$$

Now, let us write $e_{I}^{t}$ with $d\left(I_{1}, I\right) \leq s_{2}$, in the basis $e_{J}$ with $J \in \Lambda$. For any $I \in \Lambda$ we have

$$
\begin{aligned}
e_{I}^{t} & =e_{I}+t \sum_{J \in \Delta(I, 1)} \operatorname{sign}(J) e_{J}+\cdots+t_{I}^{s_{I}^{+}} \sum_{J \in \Delta\left(I, s_{I}^{+}\right)} \operatorname{sign}(J) e_{J} \\
& =\sum_{l=0}^{s_{I}^{+}}\left(t^{l} \sum_{J \in \Delta(I, l)} \operatorname{sign}(J) e_{J}\right)=\sum_{J \in \Delta(I)^{+}} t^{d(J, I)} \operatorname{sign}(J) e_{J} .
\end{aligned}
$$

where $\operatorname{sign}(J)= \pm 1$. Note that $\operatorname{sign}(J)$ depends on $J$ but not on $I$, then we can write $e_{I}^{t}=\sum_{J \in \Delta(I)^{+}} t^{d(J, I)} e_{J}$. Therefore, we have

$$
T_{t}=\left\langle e_{I}\left|d\left(I_{1}, I\right) \leq s_{1} ; \sum_{J \in \Delta(I)^{+}} t^{d(J, I)} e_{J}\right| d\left(I_{1}, I\right) \leq s_{2}\right\rangle
$$

Finally, we define

$$
\Delta:=\left\{I: d\left(I_{1}, I\right) \leq s_{1}\right\} \bigcup\left(\bigcup_{d\left(I_{1}, I\right) \leq s_{2}} \Delta(I)^{+}\right) \subset \Lambda
$$

Let $I \in \Lambda$ be an index such that $d\left(I_{1}, I\right)>s_{1}+s_{2}+1$. If $I \notin \Delta$ then $T_{t} \subset\left\{Z_{I}=0\right\}$ for any $t \neq 0$ and we are done.

Assume that $I \in \Delta$. For any $e_{K}^{t}$ with non-zero coordinate $Z_{I}$ we have $I \in \Delta(K)^{+}$, that is $K \in \Delta(I)^{-}$. Now, it is enough to find a hyperplane $H_{I}$ of type

$$
F_{I}=\sum_{J \in \Delta(I)^{-}} t^{d(J, I)} c_{J} Z_{J}=0
$$

with $c_{J} \in \mathbb{C}$ and $c_{I} \neq 0$, and such that $T_{t} \subset H_{I}$ for each $t \neq 0$. In the following, let us write $s_{i, I}^{-}=s$. Now, let us check what conditions we get by requiring $T_{t} \subset\left\{F_{I}=0\right\}$ for $t \neq 0$. Given $K \in \Delta(I)^{-}$we have that $d(I, K) \leq s_{K}^{+}$and

$$
\begin{aligned}
F_{I}\left(e_{K}^{t}\right) & =F_{I}\left(\sum_{J \in \Delta(K)^{+}} t^{d(J, K)} e_{J}\right)=\sum_{J \in \Delta(I)^{-}} t^{d(J, I)} c_{J}\left(\sum_{J \in \Delta(K)^{+}} t^{d(J, K)} e_{J}\right) \\
& =\sum_{J \in \Delta(I)^{-} \cap \Delta(K)^{+}} t^{d(J, I)+d(J, K)} c_{J}=t^{d(I, K)}\left[\sum_{J \in \Delta(I)^{-} \cap \Delta(K)^{+}} c_{J}\right]
\end{aligned}
$$


Therefore,

$$
F_{I}\left(e_{K}^{t}\right)=0 \forall t \neq 0 \Leftrightarrow \sum_{J \in \Delta(I)^{-} \cap \Delta(K)^{+}} c_{J}=0
$$

Note that this is a linear condition on the coefficients $c_{J}$, with $J \in \Delta(I)^{-}$. Hence

$$
\begin{aligned}
T_{t} \subset\left\{F_{I}=0\right\} \text { for } t \neq 0 & \Leftrightarrow \begin{cases}F_{I}\left(e_{K}\right)=0 & \forall K \in \Delta(I)^{-} \cap B\left[I_{1}, s_{1}\right] \\
F_{I}\left(e_{K}^{t}\right)=0 & \forall K \in \Delta(I)^{-} \cap B\left[I_{1}, s_{2}\right]\end{cases} \\
& \Leftrightarrow\left\{\begin{array}{ccc}
c_{K}=0 & \forall K \in \Delta(I)^{-} \cap B\left[I_{1}, s_{1}\right] \\
\sum_{J \in \Delta(I)^{-} \cap \Delta(K)^{+}} c_{J}=0 & \forall K \in \Delta(I)^{-} \cap B\left[I_{1}, s_{2}\right]
\end{array}\right.
\end{aligned}
$$

where $B[J, l]:=\{K \in \Lambda \mid d(J, K) \leq l\}$. The number of conditions on the $c_{J}$ is then $c:=\left|\Delta(I)^{-} \cap B\left[I_{1}, s_{1}\right]\right|+$ $\left|\Delta(I)^{-} \cap B\left[I_{1}, s_{2}\right]\right|$.

The problem is now reduced to find a solution of the linear system given by the $c$ equations (2.13) in the $\left|\Delta(I)^{-}\right|$variables $c_{J}, J \in \Delta(I)^{-}$such that $c_{I} \neq 0$. Therefore, it is enough to find $s+1$ complex numbers $c_{I}=c_{0} \neq 0, c_{1}, \ldots, c_{s}$ satisfying the following conditions

$$
\begin{cases}c_{j}=0 & \forall j=s, \ldots, d-s_{1} \\ \sum_{m=0}^{d(I, K)}\left|\Delta(I)^{-} \cap \Delta(K, l)\right| c_{d(I, K)-m}=0 & \forall K \in \Delta(I)^{-} \cap B\left[I_{1}, s_{2}\right]\end{cases}
$$

where $d=d\left(I_{1}, I\right)>s_{1}+s_{2}+1$. Note that (2.14) can be written as

$$
\begin{cases}c_{j}=0 & \forall j=s, \ldots, d-s_{1} \\
\sum_{m=0}^{j}\left(\begin{array}{c}
j \\
j-m
\end{array}\right) c_{m}=0 & \forall j=s, \ldots, d-s_{2}\end{cases}
$$

that is

$$
\left\{\begin{array} { l } 
{ c _ { s } = 0 } \\
{ \vdots } \\
{ c _ { d - s _ { 1 } } = 0 }
\end{array} \left\{\begin{array}{l}
\left(\begin{array}{l}
s \\
0
\end{array}\right) c_{s}+\left(\begin{array}{l}
s \\
1
\end{array}\right) c_{s-1}+\cdots+\left(\begin{array}{c}
s \\
s-1
\end{array}\right) c_{1}+\left(\begin{array}{l}
s \\
s
\end{array}\right) c_{0}=0 \\
\vdots \\
\left(\begin{array}{c}
d-s_{2} \\
0
\end{array}\right) c_{d-s_{2}}+\left(\begin{array}{c}
d-s_{2} \\
1
\end{array}\right) c_{d-s_{2}-1}+\cdots+\left(\begin{array}{c}
d-s_{2} \\
d-s_{2}-1
\end{array}\right) c_{1}+\left(\begin{array}{c}
d-s_{2} \\
d-s_{2}
\end{array}\right) c_{0}=0
\end{array}\right.\right.
$$

Now, it is enough to show that the linear system (2.15) admits a solution with $c_{0} \neq 0$. If, $s<d-s_{2}$ then the system (2.15) reduces to $c_{s}=\cdots=c_{d-s_{1}}=0$ and then we can take $c_{0}=1$ and $c_{1}=\cdots=c_{s}=0$, since $d-s_{1}>s_{2}+1>1$.

So, let us assume that $s \geq d-s_{2}$. Since $c_{s}=\cdots=c_{d-s_{1}}=0$ our problem is translated into checking that the system (2.15) admits a solution involving the variables $c_{d-s_{1}-1}, \ldots, c_{0}$ with $c_{0} \neq 0$. First of all, note that the system (2.15) can be rewritten as follows

$$
\left\{\begin{array}{l}
\left(\begin{array}{c}
s \\
s-\left(d-s_{1}-1\right)
\end{array}\right) c_{d-s_{1}-1}+\left(\begin{array}{c}
s \\
s-\left(d-s_{2}-2\right)
\end{array}\right) c_{d-s_{1}-2}+\cdots+\left(\begin{array}{c}
s \\
s-1
\end{array}\right) c_{1}+\left(\begin{array}{l}
s \\
s
\end{array}\right) c_{0}=0 \\
\vdots \\
\left(\begin{array}{c}
d-s_{2} \\
d-s_{2}-\left(d-s_{1}-1\right)
\end{array}\right) c_{d-s_{1}-1}+\left(\begin{array}{c}
d-s_{2} \\
d-s_{2}-\left(d-s_{1}-2\right)
\end{array}\right) c_{d-s_{1}-2}+\cdots+\left(\begin{array}{c}
d-s_{2} \\
d-s_{2}-1
\end{array}\right) c_{1}+\left(\begin{array}{c}
d-s_{2} \\
d-s_{2}
\end{array}\right) c_{0}=0
\end{array}\right.
$$

Thus, it is enough to check that the $\left(s-d+s_{2}+1\right) \times\left(d-s_{1}-1\right)$ matrix

$$
M=\left(\begin{array}{cccc}
\left(\begin{array}{c}
s \\
s-\left(d-s_{1}-1\right)
\end{array}\right) & \left(\begin{array}{c}
s \\
s-\left(d-s_{1}-2\right)
\end{array}\right) & \ldots & \left(\begin{array}{c}
s \\
s-1
\end{array}\right) \\
\vdots & \vdots & \ddots & \vdots \\
\left(\begin{array}{c}
d-s_{2} \\
d-s_{2}-\left(d-s_{1}-1\right)
\end{array}\right) & \left(\begin{array}{c}
d-s_{2} \\
d-s_{2}-\left(d-s_{1}-2\right)
\end{array}\right) & \ldots & \left(\begin{array}{c}
d-s_{2} \\
d-s_{2}-1
\end{array}\right)
\end{array}\right)
$$

has maximal rank. Now, note that $s \leq d$ and $d>s_{1}+s_{2}+1$ yield $s-d+s_{2}+1<s-s_{1} \leq d-s_{1}$ and then $s-d+s_{2}+1 \leq d-s_{1}-1$. Therefore, we have to show that the $\left(s-d+s_{2}+1\right) \times\left(s-d+s_{2}+1\right)$ submatrix

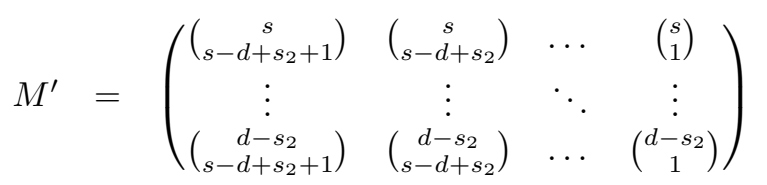

has non-zero determinant. Finally, since $d-s_{2}>s_{1}+1 \geq 1$ [GV85, Corollary 2] yields that $\operatorname{det}\left(M^{\prime}\right) \neq 0$.

Proposition 2.16. Set $\alpha=\left\lfloor\frac{n+1}{k_{r}+1}\right\rfloor$. Then, the variety $\prod_{i=1}^{r} \mathbb{G}\left(k_{i}, n\right)$ has $\alpha$-osculating regularity. 
Proof. First of all, note that if $\alpha=2$ then the statement follows form Proposition 2.12, Then we may assume $\alpha \geq 3$.

Let $p_{1}, \ldots, p_{\alpha} \in \prod_{i=1}^{r} \mathbb{G}\left(k_{i}, n\right)$ be general points. We may assume that $p_{j}=e_{I_{j}}$ for $j=1, \ldots, \alpha$. Each $e_{I_{j}}$, $j \geq 2$, is connected to $e_{I_{1}}$ by the degree $r+\sum_{i=1}^{r} k_{i}$ rational normal curve defined by

$$
\gamma_{j}(s: t)=\prod_{i=1}^{r}\left(s e_{0}+t e_{\left(k_{r}+1\right)(j-1)}\right) \wedge \cdots \wedge\left(s e_{k_{i}}+t e_{\left(k_{r}+1\right)(j-1)+k_{i}}\right)
$$

We work on the affine chart $s=1$ and set $t=(1: t)$. Now, given $s \geq 0$ we consider the family of linear subspaces

$$
T_{t}=\left\langle T_{e_{I_{1}}}^{s}, T_{\gamma_{2}(t)}^{s}, \ldots, T_{\gamma_{\alpha}(t)}^{s}\right\rangle, t \in \mathbb{P}^{1} \backslash\{0\}
$$

Our goal is to show that the flat limit $T_{0}$ of $\left\{T_{t}\right\}_{t \in \mathbb{P}^{1} \backslash\{0\}}$ in $\mathbb{G}\left(\operatorname{dim}\left(T_{t}\right), N\right)$ is contained in $T_{e_{I_{1}}}^{2 s+1}$. In order to do this, let us consider the points

$$
e_{0}, \ldots, e_{n}, e_{0}^{j, t}=e_{0}+t e_{\left(k_{r}+1\right)(j-1)}, \ldots, e_{k_{r}}^{j, t}=e_{k_{r}}+t e_{\left(k_{r}+1\right) j-1}, e_{k_{r}+1}^{j, t}=e_{k_{r}+1}, \ldots, e_{n}^{j, t}=e_{n}
$$

and, for each $I=\left\{I^{1}, \ldots, I^{r}\right\} \in \Lambda$ and $j=2, \ldots, \alpha$, the corresponding points in $e_{I}^{j, t}=e_{I^{1}}^{j, t} \otimes e_{I^{2}}^{j, t} \otimes \cdots \otimes e_{I^{r}}^{j, t} \in \mathbb{P}^{N}$. By Proposition 2.2 we have

$$
T_{t}=\left\langle e_{I}\left|d\left(I_{1}, I\right) \leq s ; e_{I}^{j, t}\right| d\left(I_{j}, I\right) \leq s, j=2, \ldots, \alpha\right\rangle, t \neq 0
$$

and

$$
T_{e_{I_{1}}}^{2 s+1}=\left\langle e_{J} \mid d\left(I_{1}, J\right) \leq 2 s+1\right\rangle=\left\{Z_{J}=0 \mid d\left(I_{1}, J\right)>2 s+1\right\}
$$

In order to show that $T_{0} \subset T_{p}^{2 s+1}$, it is enough to exhibit, for each index $I \in \Lambda$ with $d\left(I_{1}, I\right)>2 s+1$, an hyperplane $H_{I}$ of type $Z_{I}+t\left(\sum_{J \neq I} f_{J}(t) Z_{J}\right)=0$ such that $T_{t} \subset\left\{H_{i}=0\right\}$ for $t \neq 0$.

For each $l \geq 0, j=2, \ldots, \alpha$ and $I=\left\{I^{1}, \ldots, I^{r}\right\} \in \Lambda$ we define

$$
\Delta(I, l)_{j}=\left\{\left\{\left(I^{k} \backslash J^{k}\right) \cup\left(J^{k}+(j-1)\left(k_{r}+1\right)\right\}_{1 \leq k \leq r} \mid J^{k} \subset I^{k} \cap I_{1}^{k} \text { and } \sum\left|J^{k}\right|=l\right\} \subset \Lambda\right.
$$

where $L+\lambda=\{i+\lambda \mid i \in L\}$ is the translation of the set $L$ by the integer $\lambda$. For any $l>0$ we define

$$
\begin{gathered}
\Delta(I,-l)_{j}=\left\{J \mid I \in \Delta(J, l)_{j}\right\} \\
s_{I, j}^{+}=\max _{l \geq 0}\left\{\Delta(I, l)_{j} \neq \emptyset\right\} \in\left\{0, \ldots, \sum k_{j}+r\right\} \\
s_{I, j}^{-}=\max _{l>0}\left\{\Delta(I,-l)_{j} \neq \emptyset\right\} \in\left\{0, \ldots, \sum k_{j}+r\right\} \\
\Delta(I)_{j}^{+}=\bigcup_{0 \leq l} \Delta(I, l)_{j}=\bigcup_{0 \leq l \leq s_{I, j}^{+}} \Delta(I, l)_{j} \\
\Delta(I)_{j}^{-}=\bigcup_{0 \leq l} \Delta(I,-l)_{j}=\bigcup_{0 \leq l \leq s_{I, j}^{-}} \Delta(I,-l)_{j}
\end{gathered}
$$

Note that for any $l$ we have

$$
J \in \Delta(I, l)_{j} \Rightarrow d(J, I)=|l| \text { and } d\left(J, I_{1}\right)=d\left(I, I_{1}\right)+l
$$

We will write $e_{I}^{t}$ with $d\left(I_{1}, I\right) \leq s$, in the basis $e_{J}$ with $J \in \Lambda$. For any $I \in \Lambda$ we have

$$
\begin{aligned}
e_{I}^{j, t} & =e_{I}+t \sum_{J \in \Delta(I, 1)_{j}} \operatorname{sign}(J) e_{J}+\cdots+t^{s_{I, j}^{+}} \sum_{J \in \Delta\left(I, s_{I, j}^{+}\right)} \operatorname{sign}(J) e_{J} \\
& =\sum_{l=0}^{s_{I, j}^{+}}\left(t^{l} \sum_{J \in \Delta(I, l)_{j}} \operatorname{sign}(J) e_{J}\right)=\sum_{J \in \Delta(I)_{j}^{+}} t^{d(J, I)} \operatorname{sign}(J) e_{J} .
\end{aligned}
$$

where $\operatorname{sign}(J)= \pm 1$. Note that $\operatorname{sign}(J)$ depends on $J$ but not on $I$, then we can write $e_{I}^{j, t}=\sum_{J \in \Delta(I)_{j}^{+}} t^{d(J, I)} e_{J}$. Therefore, we have

$$
T_{t}=\left\langle e_{I}\left|d\left(I_{1}, I\right) \leq s ; \sum_{J \in \Delta(I)_{j}^{+}} t^{d(J, I)} e_{J}\right| d\left(I_{1}, I\right) \leq s, 2 \leq j \leq \alpha\right\rangle
$$


Finally we define

$$
\Delta:=\left\{I: d\left(I_{1}, I\right) \leq s\right\} \bigcup\left(\bigcup_{\substack{d\left(I_{1}, I\right) \leq s \\ 2 \leq j \leq \alpha}} \Delta(I)_{j}^{+}\right) \subset \Lambda
$$

Let $I \in \Lambda$ be an index such that $d\left(I_{1}, I\right)>2 s+1$. If $I \notin \Delta$, then $T_{t} \subset\left\{Z_{I}=0\right\}$ for any $t \neq 0$ and we are done.

Now, assume that $I \in \Delta$. We will show that $\Delta\left(K_{1}\right)_{j_{1}}^{+} \cap \Delta\left(K_{2}\right)_{j_{2}}^{+}=\emptyset$ whenever $K_{1}, K_{2} \in \Lambda$ with $d\left(K_{1}, I_{1}\right), d\left(K_{2}, I_{2}\right) \leq$ $s$ and $2 \leq j_{1}, j_{2} \leq \alpha$ with $j_{1} \neq j_{2}$.

In fact, suppose that $\Delta\left(K_{1}\right)_{j_{1}}^{+} \cap \Delta\left(K_{2}\right)_{j_{2}}^{+} \neq \emptyset$, that is there exists $I \in \Lambda$ such that

$$
I \in \Delta\left(K_{1}, l_{1}\right)_{j_{1}} \cap \Delta\left(K_{2}, l_{2}\right)_{j_{2}} \text { for some } l_{1} \text { and } l_{2}
$$

Now, consider the following sets

$$
\begin{aligned}
I^{0} & :=I \cap I_{1} \\
I^{1} & :=I \cap\left\{K_{1}+\left(j_{1}-1\right)\left(k_{r}+1\right)\right\} \\
I^{2} & :=I \cap\left\{K_{2}+\left(j_{2}-1\right)\left(k_{2}+1\right)\right\} \\
I^{3} & :=I \backslash\left(I^{0} \cup I^{1} \cup I^{2}\right)
\end{aligned}
$$

Since $I \in \Delta\left(K_{1}, l_{1}\right)_{j_{1}} \cap \Delta\left(K_{2}, l_{2}\right)_{j_{2}}$ we have $\left|I^{1}\right|=l_{1}$ and $\left|I^{2}\right|=l_{2}$. Set $\left|I^{3}\right|=u$, then

$$
d\left(I, I_{1}\right)=l_{1}+l_{2}+u \leq l_{1}+l_{2}+2 u \stackrel{2.17}{=} d\left(K_{1}, I_{1}\right)+d\left(K_{1}, I_{1}\right) \leq 2 s
$$

contradicting $d\left(I_{1}, I\right)>2 s+1$. Therefore we conclude that there is a unique $j_{I}$ for which

$$
I \in \bigcup_{d\left(I_{1}, I\right) \leq s} \Delta(I)_{j_{I}}^{+}
$$

Now, let $J \in \Lambda$ such that $d\left(J, I_{1}\right) \leq s$ and $I \in \Delta(J)_{j_{I}}^{+}$. Note that

$$
d\left(I, I_{1}\right)-s(I)_{j_{I}}^{-} \leq d\left(I, I_{1}\right)-d(I, J)=d\left(J, I_{1}\right) \leq s \Rightarrow s+1-D+s(I)_{j_{I}}^{-}>0
$$

where $D=d\left(I, I_{1}\right)>2 s+1$. We define

$$
\Gamma(I)=\sum_{0 \leq l \leq s+1-D+s(I)_{j_{I}}^{-}} \Delta(I,-l)_{j_{I}} \subset \Gamma
$$

Our aim now is to find a hyperplane of the form

$$
H_{I}=\left\{\sum_{J \in \Gamma(I)} t^{d(J, I)} c_{J} Z_{J}=0\right\}
$$

such that $T_{t} \subset H_{I}$ and $c_{I} \neq 0$. First, note that

$$
J \in \Gamma(I) \Rightarrow J \notin \bigcup_{\substack{d\left(I_{1}, K\right) \leq s \\ 2 \leq j \leq \alpha ; j \neq j_{I}}} \Delta(K)_{j}^{+}
$$

In fact, suppose that $J \in \Delta(I,-l)_{j_{I}} \cap \Delta(K, m)_{j}$, for some $K \in \Lambda$ with $d\left(K, I_{1}\right) \leq s$, and $0 \leq j \leq s+1-D+s(I)_{j_{I}}^{-}$ with $j \neq j_{I}$. Then, since $J \in \Delta(I,-l)_{j_{I}}$ we have

$$
\left|J \cap I_{j_{I}}\right|=\left|I \cap I_{j_{I}}\right|-l \geq s(I)_{j_{I}}^{-}-l \geq D-k-1>s
$$

On the other hand, since $J \in \Delta(K, m)_{j}$ with $j \neq j_{I}$ we have

$$
\left|J \cap I_{j_{I}}\right|=\left|K \cap I_{j_{I}}\right| \leq d\left(K, I_{1}\right) \leq s
$$

which is a contradiction. Now, note that if $K \in \Lambda$ is such that $d\left(K, I_{1}\right) \leq s$ and $K \in \Gamma(I)$, then

$$
d\left(K, I_{1}\right)=d\left(I, I_{1}\right)-d(I, K)>2 s+1-\left(s+1-D+s(I)_{j_{I}}^{-}\right)>s+D-s(I)_{j_{I}}^{-}>s
$$

Thus (2.19) yields that the hyperplane $H_{I}$ given by (2.18) is such that

$$
\left\langle e_{K}\left|d\left(I_{1}, K\right) \leq s ; \sum_{J \in \Delta(K)_{j}^{+}} t^{d(J, K)} e_{J}\right| d\left(I_{1}, K\right) \leq s, 2 \leq j \leq \alpha ; j \neq j_{I}\right\rangle \subset H_{I}, t \neq 0
$$


Therefore

$$
T_{t} \subset H_{I}, t \neq 0 \Leftrightarrow\left\langle\sum_{J \in \Delta(K)_{j_{I}}^{+}} t^{d(J, K)} e_{J} \mid d\left(I_{1}, K\right) \leq s\right\rangle \subset H_{I}, t \neq 0
$$

Now, arguing as in the proof of Proposition 2.12 we get

$$
T_{t} \subset H_{I}, t \neq 0 \Leftrightarrow \sum_{J \in \Delta(K)_{j_{I}}^{+} \cap \Gamma(I)} c_{J}=0, \quad \forall K \in \Delta(I)_{j_{I}}^{-} \cap B\left[I_{1}, s\right]
$$

So, the problem is reduced to find a solution $\left(c_{J}\right)_{J \in \Gamma(I)}$ for the linear system (2.20) such that $c_{I} \neq 0$. We set $c_{J}=c_{d(I, J)}$ and reduce, as in the proof of Proposition 2.12, to the linear system

$$
\sum_{l=0}^{s+1+D-s(I)^{-}}\left(\begin{array}{c}
D-i \\
D-i-l
\end{array}\right) c_{l}, D-S(I)_{j_{I}}^{-} \leq i \leq k
$$

We have $s+2+D-s(I)_{j_{I}}^{-}$variables $c_{0}, \ldots, c_{s+1+D-s(I)_{j_{I}}^{-}}$and $s+1+D-s(I)_{j_{I}}^{-}$equations. Finally, the argument used in the last part of the proof of Proposition 2.12 shows that the linear system (2.21) admits a solution with $c_{0} \neq 0$.

\section{On SECANT Defectivity of PRoducts of Grassmannians}

Let $X \subset \mathbb{P}^{N}$ be an irreducible non-degenerate variety of dimension $n$ and let

$$
\Gamma_{h}(X) \subset X \times \cdots \times X \times \mathbb{G}(h-1, N)
$$

where $h \leq N$, be the closure of the graph of the rational map $\alpha: X \times \cdots \times X-\rightarrow \mathbb{G}(h-1, N)$ taking $h$ general points to their linear $\operatorname{span}\left\langle x_{1}, \ldots, x_{h}\right\rangle$. Observe that $\Gamma_{h}(X)$ is irreducible and reduced of dimension $h n$.

Let $\pi_{2}: \Gamma_{h}(X) \rightarrow \mathbb{G}(h-1, N)$ be the natural projection, and $\mathcal{S}_{h}(X):=\pi_{2}\left(\Gamma_{h}(X)\right) \subset \mathbb{G}(h-1, N)$. Again $\mathcal{S}_{h}(X)$ is irreducible and reduced of dimension $h n$. Finally, consider

$$
\mathcal{I}_{h}=\{(x, \Lambda) \mid x \in \Lambda\} \subset \mathbb{P}^{N} \times \mathbb{G}(h-1, N)
$$

with natural projections $\pi_{h}$ and $\psi_{h}$ onto the factors.

The abstract $h$-secant variety is the irreducible variety $\operatorname{Sec}_{h}(X):=\left(\psi_{h}\right)^{-1}\left(\mathcal{S}_{h}(X)\right) \subset \mathcal{I}_{h}$. The h-secant variety is $\operatorname{Sec}_{h}(X):=\pi_{h}\left(\operatorname{Sec}_{h}(X)\right) \subset \mathbb{P}^{N}$. Then $\operatorname{Sec}_{h}(X)$ is an $(h n+h-1)$-dimensional variety.

The number $\delta_{h}(X)=\min \{h n+h-1, N\}-\operatorname{dim} \operatorname{Sec}_{h}(X)$ is called the $h$-secant defect of $X$. We say that $X$ is $h$-defective if $\delta_{h}(X)>0$. We refer to Rus03 for a comprehensive survey on the subject.

Determining secant defectivity is a classical problem in algebraic geometry. A new strategy to determine the non secant defectivity was introduced in [MR19, Theorem 5.3], the method is based on degenerating the span of several tangent spaces $T_{x_{i}} X$ in a single osculating space $T_{x}^{s} X$.

To state the criterion for non secant defectivity in [MR19] we introduce a function $h_{m}: \mathbb{N}_{\geq 0} \longrightarrow \mathbb{N}_{\geq 0}$ counting how many tangent spaces can be degenerated into a higher order osculating space.

Definition 3.1. Given an integer $m \geq 0$ we define a function

$$
h_{m}: \mathbb{N}_{\geq 0} \longrightarrow \mathbb{N}_{\geq 0}
$$

as follows: for $h_{m}(0)=0$ and for any $k>0$ write

$$
k+1=2^{\lambda_{1}}+2^{\lambda_{2}}+\cdots+2^{\lambda_{l}}+\varepsilon
$$

where $\lambda_{1}>\lambda_{2}>\cdots>\lambda_{l} \geq 1$ and $\varepsilon \in\{0,1\}$, then

$$
h_{m}(k)=m^{\lambda_{1}-1}+m^{\lambda_{2}-1}+\cdots+m^{\lambda_{l}-1}
$$

Theorem 3.2. [MR19, Theorem 5.3] Let $X \subset \mathbb{P}^{N}$ be a projective variety having m-osculating regularity and strong 2 -osculating regularity. Let $s_{1}, \ldots, s_{l} \geq 1$ integers such that the general osculating projection $\Pi_{p_{1}, \ldots, p_{l}}^{s_{1}, \ldots, s_{l}}$ is generically finite. If

$$
h \leq \sum_{j=1}^{l} h_{m}\left(s_{j}\right)
$$

then $X$ is not $(h+1)$-defective.

Now, we are ready to prove our main result on non-defectivity of product of Grassmannians. We follow the notation introduced in the previous sections. 
Theorem 3.3. Assume that $n \geq 2 k_{r}+1$. Set

$$
\alpha:=\left\lfloor\frac{n+1}{k_{r}+1}\right\rfloor
$$

and let $h_{\alpha}$ be as in Definition 3.1. Assume that

- either $n \geq k_{r}^{2}+3 k_{r}+1$ and $h \leq \alpha h_{\alpha}\left(\sum_{i=1}^{r} k_{i}+r-2\right)$ or

- $\alpha\left(k_{r}+1\right)-1<n<k_{r}^{2}+3 k_{r}+1$ and $h \leq(\alpha-1) h_{\alpha}\left(\sum_{i=1}^{r} k_{i}+r-2\right)+h_{\alpha}\left(s^{\prime}\right)$

where $s^{\prime}=\sum_{i=1}^{r} s_{i}-2$ with $s_{i}^{\prime}=\min \left\{k_{i}+1, n-\alpha\left(k_{i}+1\right)\right\}$ for $i \neq r$ and $s_{r}^{\prime}=\min \left\{k_{r}, n-\alpha k_{r}-1\right\}$. Then $\prod_{i=1}^{r} \mathbb{G}\left(k_{i}, n\right)$ is not $(h+1)$-defective.

Proof. We have shown in Propositions 2.16, 2.12 that $\prod_{i=1}^{r} \mathbb{G}\left(k_{i}, n\right)$ has respectively $\alpha$-osculating regularity for $\alpha:=\left\lfloor\frac{n+1}{k_{r}+1}\right\rfloor$, and strong 2 -osculating regularity. The statement then follows immediately from Proposition 2.10 and Theorem 3.2

Corollary 3.4. The variety $\prod_{i=1}^{r} \mathbb{G}\left(k_{i}, n\right)$ is not $(h+1)$-defective for $h \leq\left(\frac{n+1}{k_{r}+1}\right)^{\left\lfloor\log _{2}\left(\sum k_{j}+r-1\right)\right\rfloor}$.

Proof. We may write

$$
\sum_{i=1}^{r} k_{i}+r-1=2^{\lambda_{1}}+2^{\lambda_{2}}+\cdots+2^{\lambda_{l}}+\varepsilon
$$

with $\lambda_{1}>\lambda_{2}>\cdots>\lambda_{l} \geq 1$ and $\varepsilon \in\{0,1\}$. Then $h_{\alpha}\left(\sum_{i=1}^{r} k_{i}+r-2\right)=\alpha^{\lambda_{1}-1}+\alpha^{\lambda_{2}-1}+\cdots+\alpha^{\lambda_{l}-1}$.

The first bound in Theorem 3.3 gives $h \leq \alpha^{\lambda_{1}}+\cdots+\alpha^{\lambda_{l}}$. Furthermore, considering just the first summand in the second bound in Theorem 3.3 we get that $\prod_{i=1}^{r} \mathbb{G}\left(k_{i}, n\right)$ is not $(h+1)$-defective for $h \leq(\alpha-1)\left(\alpha^{\lambda_{1}-1}+\right.$ $\left.\alpha^{\lambda_{2}-1}+\cdots+\alpha^{\lambda_{l}-1}\right)$.

Finally, from (3.5) we get that $\lambda_{1}=\left\lfloor\log _{2}\left(r-1+\sum k_{i}\right)\right\rfloor$. Hence, asymptotically we have $h_{\alpha}\left(\sum k_{j}+r-2\right) \sim$ $\alpha^{\left\lfloor\log _{2}\left(r-1+\sum k_{i}\right)\right\rfloor-1}$, and by Theorem 3.3 if $h \leq\left(\frac{n+1}{k_{r}+1}\right)^{\left\lfloor\log _{2}\left(\sum k_{j}+r-1\right)\right\rfloor}$ then the variety $\prod_{i=1}^{r} \mathbb{G}\left(k_{i}, n\right)$ is not $(h+1)$-defective.

\section{ON SECANT DEFECTIVITY OF FLAG VARIETIES}

Our goal is to compute the higher osculating spaces of $\mathbb{F}\left(k_{1}, \ldots, k_{r} ; n\right)$. In order to do this, we will use the following notion introduced in [FMR18, Definition 3.2].

Definition 4.1. Let $X \subset \mathbb{P}^{N}$ be an irreducible variety and $Y=\mathbb{P}^{k} \cap X$ be a linear section of $X$. We say that $Y$ is osculating well-behaved if for each smooth point $p \in Y$ we have

$$
T_{p}^{s} Y=\mathbb{P}^{k} \cap T_{p}^{s} X
$$

for every $s \geq 0$.

Let us denote by $M_{i}$ the following $\left(k_{i}+1\right) \times(n+1)$ matrix

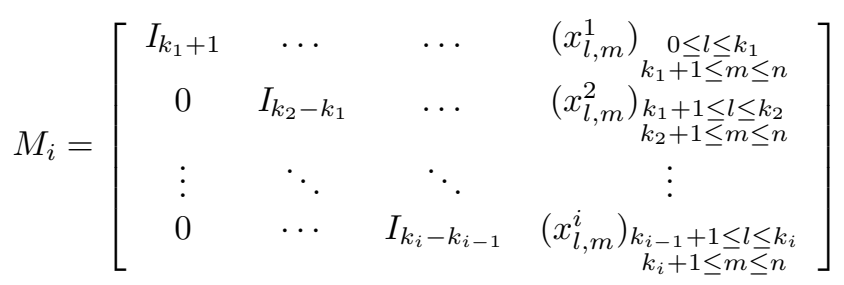

and consider the map

$$
\begin{aligned}
& \varphi^{\prime}: \prod_{i=1}^{r} \mathbb{C}^{\left(k_{1}+1\right)\left(n-k_{1}\right)+\sum_{j=2}^{i}\left(n-k_{j}\right)\left(k_{j}-k_{j-1}\right)} \longrightarrow \mathbb{P}^{N} \\
&\left(M_{1}, \ldots, M_{r}\right) \longmapsto \\
&\left.\prod_{i=1}^{r} \operatorname{det}\left(M_{J^{i}}\right)\right)_{J=\left\{J^{1}, \ldots, J^{r}\right\} \in \Lambda}
\end{aligned}
$$

where $M_{J^{i}}$ is the submatrix obtained from $M_{i}$ by considering only the columns indexed by $J^{i}$.

For each $2 \leq i \leq r$ and $m \leq k_{l}$, let us take $x_{l, m}^{i}=0$ in $M_{i}$. Then $\varphi^{\prime}$ becomes the parametrization $\varphi$ of $\prod_{i=1}^{r} \mathbb{G}\left(k_{i}, n\right)$ in $(2.3)$. 
Now, set $x_{l, m}^{i}=x_{l, m}^{r}$ in $M_{i}$ for each $i=1, \ldots, r-1$ and $1 \leq l<m \leq n$. Hence $\varphi$ becomes the parametrization of $\mathbb{F}\left(k_{1}, \ldots, k_{r} ; n\right)$ given by

$$
\begin{aligned}
& \bar{\varphi}: \quad \mathbb{C}^{\left(k_{1}+1\right)\left(n-k_{1}\right)+\sum_{j=2}^{r}\left(n-k_{j}\right)\left(k_{j}-k_{j-1}\right)} \longrightarrow \mathbb{P}\left(\Gamma_{a}\right) \subset \mathbb{P}^{N} \\
& M_{r} \quad \longmapsto \varphi\left(\bar{M}_{1}, \ldots, \bar{M}_{r}\right)
\end{aligned}
$$

where $\bar{M}_{i}$ is the submatrix obtained from $M_{r}$ by considering only the first $k_{i}+1$ rows.

Lemma 4.2. Let $T_{\varphi^{\prime}}^{s}\left(\prod_{i=1}^{r} \mathbb{G}\left(k_{i}, n\right)\right):=\left\langle\frac{\partial^{|I|} \varphi^{\prime}}{\partial x_{|I|}}(0)|| I \mid \leq s\right\rangle$ be the s-osculating space of $\prod_{i=1}^{r} \mathbb{G}\left(k_{i}, n\right)$ with respect to $\varphi^{\prime}$. Then $T_{\varphi^{\prime}}^{s}\left(\prod_{i=1}^{r} \mathbb{G}\left(k_{i}, n\right)\right)=T^{s}\left(\prod_{i=1}^{r} \mathbb{G}\left(k_{i}, n\right)\right)$ for every $s \leq r+\sum_{i=1}^{r} k_{i}$. In particular,

$$
\frac{\partial^{s} \varphi^{\prime}}{\partial x_{|I|}}(0)=\frac{\partial^{|J|} \varphi}{\partial x_{|J|}}(0)
$$

for some $J$ with $|J| \leq|I|$.

Proof. First, note that if for any $x_{l, m}^{i} \in x_{|I|}$ we have $m>k_{i}$, then $\frac{\partial^{s} \varphi^{\prime}}{\partial x_{|I|}}(0)=\frac{\partial^{|I|} \varphi}{\partial x_{|I|}}(0)$ and we are done.

Now, let $2 \leq i \leq r$ and consider a derivative $\frac{\partial^{|I|} \varphi^{\prime}}{\partial x_{|I|}}(0)$ such that $x_{l, m}^{i} \in x_{|I|}$ with $m \leq k_{i}$. Therefore, to prove the statement it is enough to show that this partial derivative can be written in terms of another partial derivative $\frac{\partial^{|J|} \varphi^{\prime}}{\partial x_{|J|}}(0)$ with $x_{l, m}^{i} \notin x_{|J|}, m \leq k_{i}$ and $|J|<|I|$.

Fix $2 \leq i \leq r$ and let $x_{l_{1}, m_{1}}^{i}, \ldots, x_{l_{h}, m_{h}}^{i}, x_{l_{h+1}, m_{h+1}}^{i}, \ldots, x_{l_{b}, m_{b}}^{i} \in x_{|I|}$ such that $m_{a} \leq k_{i}$ for every $a=1, \ldots, h$ and $b \leq k_{i}+1$.

If $\frac{\partial^{b} \varphi^{\prime}}{\partial x_{l_{1}, m_{1}}^{i} \cdots \partial x_{l_{h}, m_{h}}^{2} \partial x_{l_{h+1}, m_{h+1}}^{i} \cdots \partial x_{l_{b}, m_{b}}^{i}}(0) \neq 0$ consider the minor $M_{J^{i}}$ of $M_{i}$ such that the monomial

$$
x_{l_{1}, m_{1}}^{i} \ldots x_{l_{h}, m_{h}}^{i} x_{l_{h+1}, m_{h+1}}^{i} \ldots x_{l_{b}, m_{b}}^{i}
$$

appears in the expression of $\operatorname{det}\left(M_{J^{i}}\right)$. Then, there exist variables $x_{\sigma_{J^{i}}\left(l_{h+1}\right), \sigma_{J^{i}}\left(m_{h+1}\right)}^{i}, \ldots, x_{\sigma_{J^{i}}}^{i}\left(l_{b}\right), \sigma_{J^{i}}\left(m_{b}\right)$ such that $x_{\sigma_{J^{i}}\left(l_{h+1}\right), \sigma_{J^{i}}\left(m_{h+1}\right)} \cdots x_{\sigma_{J^{i}}\left(l_{b}\right), \sigma_{J^{i}}\left(m_{b}\right)}^{i}$ is also a monomial in $\operatorname{det}\left(M_{J}\right)$, where $\sigma_{J^{i}}$ is a permutation on the indexes such that $\sigma_{J^{i}}\left(m_{a}\right)>k_{i}$ for all $h+1 \leq a \leq b$.

This shows that

$$
\frac{\partial^{m} \varphi^{\prime}}{\partial x_{l_{1}, m_{1}}^{i} \cdots \partial x_{l_{h}, m_{h}}^{i} \partial x_{l_{h+1}, m_{h+1}}^{i} \cdots \partial x_{l_{b}, m_{b}}^{i}}(0)=\frac{\partial^{m} \varphi^{\prime}}{\partial x_{\sigma_{J^{i}}\left(l_{h+1}\right), \sigma_{J^{i}}\left(m_{h+1}\right)}^{i}, \ldots, \partial x_{\sigma_{J^{i}}\left(l_{b}\right), \sigma_{J^{i}}\left(m_{b}\right)}^{i}}(0)
$$

We have thus decreased the number of variables with respect we differentiate and thus lowered the order of the derivatives. Finally, since $\frac{\partial \varphi}{\partial x_{l, m}^{i}}(0)=\frac{\partial \varphi^{\prime}}{\partial x_{l, m}^{i}}(0)$ for $m>k_{i}$ we are done.

Lemma 4.3. Since $\bar{\varphi}$ is a sub-parametrization of $\varphi^{\prime}$ by the chain rule we have

$$
\frac{\partial^{s} \bar{\varphi}}{\partial x_{|I|}}(0)=\sum_{|K|} \frac{\partial^{s} \varphi^{\prime}}{\partial x_{|K|}}(0)=\sum_{|J|} \frac{\partial^{s} \varphi}{\partial x_{|J|}}(0)
$$

where $|K|=|I|=s$ and $|J| \leq|I|$. Let $\frac{\partial^{s} \bar{\varphi}}{\partial x_{|I|}}(0) \neq 0$ with $|I|=s$ such that for each $x_{l, m}^{i} \in x_{|I|}$ we have that $m>k_{i}$. Then, in the above decomposition there is at least a vector $\frac{\partial^{s} \varphi}{\partial x_{|J|}}(0)$ with $|J|=s$.

Proof. For any $x_{l, m}^{i} \in x_{|I|}$ let $h(m)$ be the maximum index in $\{1, \ldots, r\}$ such that $m>k_{h(m)}$. Since for each $x_{l, m}^{i} \in x_{|I|}$ we have that $m>k_{i}$ and $\frac{\partial^{s} \bar{\varphi}}{\partial x_{|I|}}(0) \neq 0$, we get that any $x_{l, m}^{i} \in x_{|I|}$ appears at most $h(m)$ times in $x_{|I|}$.

Now, for any $s \leq h(m)$, the chain rule expression of $\frac{\partial^{s} \bar{\varphi}}{\left(\partial x_{l, m}^{i}\right)^{s}}(0)$ contains the factor

$$
\frac{\partial^{s} \varphi^{\prime}}{\partial x_{l, m}^{1} \partial x_{l, m}^{2} \ldots \partial x_{l, m}^{h(m)}}(0)=\frac{\partial^{s} \varphi}{\partial x_{l, m}^{1} \partial x_{l, m}^{2} \ldots \partial x_{l, m}^{h(m)}}(0)
$$

Repeating this argument for all indexes $x_{l, m}^{i} \in x_{|I|}$ we conclude. 
Proposition 4.4. The flag variety is osculating well-behaved, that is

$$
T_{p}^{s} \mathbb{F}\left(k_{1}, \ldots, k_{r} ; n\right)=T_{p}^{s} \prod_{i=1}^{r} \mathbb{G}\left(k_{i}, n\right) \cap \mathbb{P}\left(\Gamma_{a}\right)
$$

for any $p \in \mathbb{F}\left(k_{1}, \ldots, k_{r} ; n\right)$ and non-negative integer $s$.

Proof. We may assume that $p=e_{I}$ where $I=\left\{I^{1}, \ldots, I^{r}\right\}$ and $I^{l}=\left\{0, \ldots, k_{l}\right\}$ for each $1 \leq l \leq r$. Let us first assume that $s=r+\sum_{i=1}^{r} k_{i}$. Note that $s$ is the smallest integer for which $T_{p}^{s} \mathbb{F}\left(k_{1}, \ldots, k_{r} ; n\right)=\mathbb{P}\left(\Gamma_{a}\right)$ and $T_{p}^{s} \prod_{i=1}^{s} \mathbb{G}\left(k_{i}, n\right)=\mathbb{P}^{N}$, in this case $T_{p}^{s} \mathbb{F}\left(k_{1}, \ldots, k_{r} ; n\right)=\mathbb{P}\left(\Gamma_{a}\right)=\mathbb{P}\left(\Gamma_{a}\right) \cap \mathbb{P}^{N}=\mathbb{P}\left(\Gamma_{a}\right) \cap T_{p}^{s} \prod_{i=1}^{s} \mathbb{G}\left(k_{i}, n\right)$ and we are done. Now, assume $s<r+\sum_{i=1}^{r} k_{i}$. Let

$$
v=\sum_{|I| \leq s-1} \alpha_{|I|} \frac{\partial^{|I|} \varphi}{\partial x_{|I|}}(0)
$$

be a general vector in $T_{p}^{s-1} \prod_{i=1}^{r} \mathbb{G}\left(k_{i}, n\right)$, and assume that

$$
v \in T_{p}^{s-1} \prod_{i=1}^{r} \mathbb{G}\left(k_{i}, n\right) \cap \mathbb{P}\left(\Gamma_{a}\right) \subset T_{p}^{s} \prod_{i=1}^{r} \mathbb{G}\left(k_{i}, n\right) \cap \mathbb{P}\left(\Gamma_{a}\right)=T_{p}^{s} \mathbb{F}\left(k_{1}, \ldots, k_{r} ; n\right)
$$

this yields that $v$ can be written as

$$
v=\sum_{|I| \leq s-1} \beta_{|I|} \frac{\partial^{|I|} \bar{\varphi}}{\partial^{|I|} x_{|I|}}(0)+\sum_{|I|=s} \beta_{|I|} \frac{\partial^{|I|} \bar{\varphi}}{\partial^{|I|} x_{|I|}}(0)
$$

Now, recall that for any $I$ such that there are variables $x_{l, m}^{i} \in x_{|I|}$ with $m \leq k_{i}$ we can find another set $J$ for which $|J|<|I|$ and

$$
\frac{\partial^{s} \varphi^{\prime}}{\partial x_{|I|}}(0)=\frac{\partial^{|J|} \varphi}{\partial x_{|J|}}(0)
$$

Therefore, we can assume that any set $I$ in the second summand of (4.6) is such that $m>k_{i}$ for any $x_{l, m}^{i} \in x_{|I|}$. Thus, by Lemma 4.3. we will have an equality in (4.5) and (4.6) if and only if $\beta_{|I|}=0$ for any set $I$ such that $m>k_{i}$ for all $x_{l, m}^{i} \in x_{|I|}$. Hence $v \in T_{p}^{s-1} \mathbb{F}\left(k_{1}, \ldots, k_{r} ; n\right)$.

4.6. Osculating Projections. Let $s_{1}, \ldots, s_{\alpha}$ be integers such that $0 \leq s_{m} \leq r-2+\sum_{i=1}^{r} k_{i}$. Denote $T_{p}^{s} \mathbb{F}\left(k_{1}, \ldots, k_{r} ; n\right)$ simply by $T_{p}^{s} \mathbb{F}$ and the linear subspace $\left\langle T_{e_{I_{1}}}^{s_{1}} \mathbb{F}, \ldots, T_{e_{I_{m}}}^{s_{m}} \mathbb{F}\right\rangle$ by $T_{e_{I_{1}}, \ldots, e_{I_{m}}}^{s_{1}, \ldots, s_{m}} \mathbb{F}$. Then, for $m \leq \alpha$ we have the linear projection

$$
\Pi_{T_{e_{I_{1}}, \ldots, e_{I_{m}} s_{1}, \ldots, s_{m}} \mathbb{F}}: \mathbb{F}\left(k_{1}, \ldots, k_{r} ; n\right) \rightarrow \mathbb{P}^{N_{s_{1}, \ldots, s m}}
$$

Proposition 4.7. Let $I_{1}, \ldots, I_{\alpha}$ be as in 2.7) and $s=r-2+\sum_{i=1}^{r} k_{i}$. Then,

- $\Pi_{T_{I_{1}, \ldots, s, e_{I_{\alpha-1}}, \ldots}, \mathbb{F}}$ is birational;

- $\Pi_{T_{e_{I_{1}}, \ldots, e_{I_{\alpha}}}^{s, \ldots, s} \mathbb{F}}$ is birational whenever $n \geq k_{r}^{2}+3 k_{r}+1$.

Proof. Since $\Pi_{T_{e_{I_{1}}, \ldots, e_{I_{\alpha-1}}}^{s, \ldots, s}}$ factors trough $\Pi_{T_{e_{I_{1}}, \ldots, e_{I_{\alpha-1}}}^{s, \ldots, s} \mathbb{F}}$, it is enough to show that the restriction of $\Pi_{T_{e_{I_{1}}, \ldots, e_{I_{\alpha-1}}}^{s, \ldots, s}}$ to $\mathbb{F}\left(k_{1}, \ldots, k_{r}\right)$ is birational.

For any $i \neq r$ and $1 \leq j \leq \alpha-1$ consider $I_{j}^{\prime i}=I_{j}^{i}$ and $I_{j}^{\prime r} \subset I_{j}^{r}$ of cardinality $k_{r}$. Since $n \geq 2 k_{r}+1$ and $k_{r} \geq k_{i}$ we must have

$$
n-\sum_{j=1}^{\alpha-1}\left|I_{j}^{\prime i}\right|=n-(\alpha-1)\left(k_{i}+1\right) \geq n-(\alpha-1) k_{r} \geq k_{r}+1 \leq k_{i}+1
$$

Now, let us denote by $I^{\prime i}$ the union $\bigcup_{j=1}^{\alpha-1} I_{j}^{\prime i}$. Then, by Lemma 2.8 there exists a rational map $\pi_{I^{\prime r}}$ making the following diagram commutative

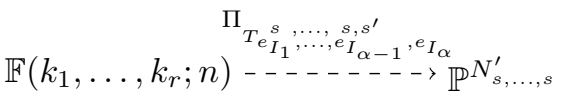

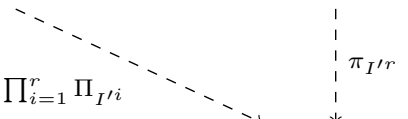

$$
\begin{aligned}
& \prod_{i=1}^{r} \mathbb{G}\left(k_{i}, n-\sum_{j=1}^{\alpha}\left|I_{j}^{\prime i}\right|\right)
\end{aligned}
$$


Now, let $x=\left(\left\{V_{i}\right\}_{i=1}^{r}\right)$ be a general point in the image of $\Pi_{T_{e_{1}, \ldots, s, e_{I_{\alpha-1}}^{s, \ldots,}}}$ and $X \subset \mathbb{F}\left(k_{1}, \ldots, k_{r} ; n\right)$ be the fiber of $\Pi_{T_{e_{1}, \ldots, \ldots, e_{I_{\alpha-1}}^{s, \ldots, s}}}$ over $x$. Set $x_{I^{\prime r}}=\pi_{I^{\prime r}}(x)$ and denote by $X_{I^{\prime r}} \subset \mathbb{F}\left(k_{1}, \ldots, k_{r} ; n\right)$ the fiber of $\prod_{i=1}^{r} \Pi_{I^{\prime i}}$ over $x_{I^{\prime r}}$.

Therefore, $X \subset \bigcap_{I^{\prime r}} X_{I^{\prime r}}$, where the intersection runs over all sets $I^{\prime r}=\bigcup_{j=1}^{\alpha-1} I_{j}^{\prime r}$ with $I_{j}^{\prime r} \subset I_{j}^{r}$ and $\left|I_{j}^{\prime r}\right|=k_{r}$ for $1 \leq j \leq \alpha-1$.

Now, note that if $\left\{W_{i}\right\}_{i=1}^{r} \in X$ is a general point, then we must have $W_{i} \subset\left\langle e_{m} \mid m \in \bigcup_{j=1}^{\alpha} I_{j}^{\prime i} ; V_{i}\right\rangle$ for any choice of $\bigcup_{j=1}^{\alpha} I_{j}^{\prime i}$. Hence,

$$
W_{i} \subset \bigcap_{I^{\prime r}}\left\langle e_{m} \mid m \in \bigcup_{j=1}^{\alpha} I_{j}^{\prime i} ; V_{i}\right\rangle
$$

In particular, $W_{r} \subset \bigcap_{I^{\prime r}}\left\langle e_{m} \mid m \in \bigcup_{j=1}^{\alpha} I_{j}^{\prime r} ; V_{r}\right\rangle$. Now, since $\left|I_{j}^{\prime r}\right| \leq k_{r}$ we must have $\bigcap_{I^{\prime r}}\left\langle e_{m} \mid m \in \bigcup_{j=1}^{\alpha} I_{j}^{\prime r}\right\rangle=\emptyset$ and then $V_{r}=\bigcap_{I^{\prime r}}\left\langle e_{m} \mid m \in \bigcup_{j=1}^{\alpha} I_{j}^{\prime r} ; V_{r}\right\rangle$ which yields $W_{r}=V_{r}$.

Now, set $i \leq r-1$. Since $\left\{V_{i}\right\}_{i \in K}$ is general in $\mathbb{F}\left(k_{1}, \ldots, k_{r} ; n\right)$ and $n-\sum_{j=1}^{\alpha-1}\left|I_{j}^{\prime i}\right| \geq k_{r}+1$ we have $V_{r} \cap\left\langle e_{m}\right| m \in$ $\left.\bigcup_{j=1}^{\alpha} I_{j}^{i}\right\rangle=\emptyset$. On the other hand $W_{i} \subset W_{r}=V_{r}$ for all $i \leq r-1$, then $W_{i} \cap\left\langle e_{m} \mid m \in \bigcup_{j=1}^{\alpha} I_{j}^{i}\right\rangle=\emptyset$. Hence, by (4.8) we must have $W_{i}=V_{i}$ for any $i \leq r-1$.

Now, assume that $n \geq k_{r}^{2}+3 k_{r}+1$ then

$$
\begin{aligned}
n-\alpha\left(k_{i}+1\right) \geq n-\alpha k_{r} & \geq n-\frac{(n+1)}{k_{r}+1} k_{r}=\frac{n\left(k_{r}+1\right)-(n+1) k_{r}}{k_{r}+1} \\
& =\frac{n-k_{r}}{k_{r}+1} \geq \frac{k_{r}^{2}+3 k_{r}+1-k_{r}}{k_{r}+1}=k_{r}+1
\end{aligned}
$$

Then, arguing as in the proof of the first case, for any choice of subsets $I_{j}^{\prime i} \subset I_{j}^{i}, I_{j}^{\prime i}=I_{j}^{i}$ with $i \neq r$ and $1 \leq j \leq \alpha-1, I_{j}^{\prime r} \subsetneq I_{j}^{r}$ of cardinality $k_{r}$ we get, by Lemma 2.8, a rational map $\pi_{I^{\prime r}}$ making the following diagram commutative

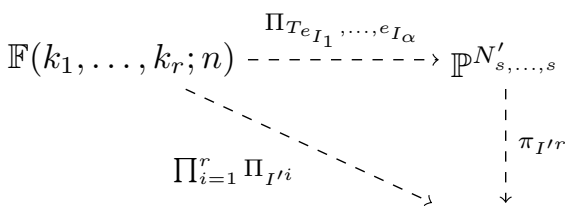

$$
\begin{aligned}
& \prod_{i=1}^{r} \mathbb{G}\left(k_{i}, n-\sum_{j=1}^{\alpha}\left|I_{j}^{\prime i}\right|\right)
\end{aligned}
$$

where $I^{\prime i}=\bigcup_{j=1}^{\alpha} I_{j}^{\prime i}, i=1, \ldots, r$. Now, to conclude it is enough to follow the same argument used in the end of the proof of the first claim.

4.8. Non-Secant defectivity of flag varieties. We recall [FMR18, Proposition 4.4] which describes how the notion of osculating regularity behaves under linear sections.

Proposition 4.9. Let $X \subset \mathbb{P}^{N}$ be an irreducible projective variety and $Y=\mathbb{P}^{k} \cap X$ a linear section of $X$ that is osculating well-behaved. Assume that given general points $p_{1}, \ldots, p_{m} \in Y$ one can find smooth curves $\gamma_{j}: C \rightarrow X, j=2, \ldots, m$, realizing the $m$-osculating regularity of $X$ for $p_{1}, \ldots, p_{m}$ such that $\gamma_{j}(C) \subset Y$. Then $Y$ has $m$-osculating regularity as well. Furthermore, the analogous statement for strong 2-osculating regularity holds as well.

Proposition 4.10. The flag variety $\mathbb{F}\left(k_{1}, \ldots, k_{r} ; n\right)$ has strong 2-osculating regularity and $\alpha$-osculating regularity, where $\alpha:=\left\lfloor\frac{n+1}{k_{r}+1}\right\rfloor$.

Proof. The statement follows immediately from Propositions 2.12, 2.16, 4.9,

Now, we are ready to prove our main result on non-defectivity of flags varieties.

Theorem 4.11. Assume that $n \geq 2 k_{r}+1$. Set

$$
\alpha:=\left\lfloor\frac{n+1}{k_{r}+1}\right\rfloor
$$


and let $h_{\alpha}$ be as in Definition 3.1. If either

- $n \geq k_{r}^{2}+3 k_{r}+1$ and $h \leq \alpha h_{\alpha}\left(\sum k_{j}+r-2\right)$ or

- $n<k_{r}^{2}+3 k_{r}+1$ and $h \leq(\alpha-1) h_{\alpha}\left(\sum k_{j}+r-2\right)$.

Then, $\mathbb{F}\left(k_{1}, \ldots, k_{r} ; n\right)$ is not $(h+1)$-defective. In particular, if

$$
h \leq\left(\frac{n+1}{k_{r}+1}\right)^{\left\lfloor\log _{2}\left(\sum k_{j}+r-1\right)\right\rfloor}
$$

then $\mathbb{F}\left(k_{1}, \ldots, k_{r} ; n\right)$ is not $(h+1)$-defective.

Proof. The first part is an immediately consequence of Propositions 4.9, 4.7 and Theorem 3.2. For the last claim note that if we write

$$
\sum k_{j}+r-1=2^{\lambda_{1}}+2^{\lambda_{2}}+\cdots+2^{\lambda_{l}}+\varepsilon
$$

with $\lambda_{1}>\lambda_{2}>\cdots>\lambda_{l} \geq 1$ and $\varepsilon \in\{0,1\}$. Then

$$
h_{\alpha}\left(\sum k_{j}+r-2\right)=\alpha^{\lambda_{1}-1}+\alpha^{\lambda_{2}-1}+\cdots+\alpha^{\lambda_{l}-1}
$$

Therefore, the first bound in Theorem 4.11 yields

$$
h \leq \alpha^{\lambda_{1}}+\alpha^{\lambda_{2}}+\cdots+\alpha^{\lambda_{l}}
$$

Furthermore, by the second bound in Theorem 4.11 we get that $\mathbb{F}\left(k_{1}, \ldots, k_{r} ; n\right)$ is not $(h+1)$-defective for

$$
h \leq(\alpha-1)\left(\alpha^{\lambda_{1}-1}+\alpha^{\lambda_{2}-1}+\cdots+\alpha^{\lambda_{l}-1}\right)
$$

Finally, by (4.12) we get that $\lambda_{1}=\left\lfloor\log _{2}\left(\sum k_{j}+r-1\right)\right\rfloor$. Hence, asymptotically we have $h_{\alpha}\left(\sum k_{j}+r-2\right) \sim$ $\alpha^{\left\lfloor\log _{2}\left(\sum k_{j}+r-1\right)\right\rfloor}$, and by Theorem 4.11 for $h \leq \alpha^{\left\lfloor\log _{2}\left(\sum k_{j}+r-1\right)\right\rfloor} \leq\left(\frac{n+1}{k_{r}+1}\right)^{\left\lfloor\log _{2}\left(\sum k_{j}+r-1\right)\right\rfloor}$ the flag variety $\mathbb{F}\left(k_{1}, \ldots, k_{r} ; n\right)$ is not $(h+1)$-defective.

Remark 4.13. Now, given a flag $\mathbb{F}\left(k_{1}, \ldots, k_{r} ; n\right)$ with $n<2 k_{r}+1$. Assume that $n \geq 2 k_{j}+1$ for some index $j$ and let $l$ be the maximum among these j's. Then we have a natural projection

$$
\begin{aligned}
\pi: \mathbb{F}\left(k_{1}, \ldots, k_{r} ; n\right) & \longrightarrow \mathbb{F}\left(k_{1}, \ldots, k_{l} ; n\right) \\
\left\{V_{i}\right\}_{i=1, \ldots, r} & \longmapsto\left\{V_{i}\right\}_{i=1, \ldots, l}
\end{aligned}
$$

The fiber of $\pi$ over a general point in $\mathbb{F}\left(k_{1}, \ldots, k_{l} ; n\right)$ is isomorphic to $\mathbb{F}\left(k_{l+1}-k_{l}-1, \ldots, k_{r}-k_{l}-1 ; n-k_{l}-1\right)$. Now let $p_{1}, \ldots, p_{h} \in \mathbb{F}\left(k_{1}, \ldots, k_{l} ; n\right)$ be general points, and $T_{p_{i}} \mathbb{F}\left(k_{1}, \ldots, k_{l} ; n\right)$ be the tangent space at $p_{i}$. Then, we have

$$
T_{\pi^{-1}\left(p_{i}\right)} \mathbb{F}\left(k_{1}, \ldots, k_{r} ; n\right)=\left\langle T_{p_{i}} \mathbb{F}\left(k_{1}, \ldots, k_{l} ; n\right), T_{\pi^{-1}\left(p_{i}\right)} \mathbb{F}\left(k_{l+1}-k_{l}, \ldots, k_{r}-k_{l} ; n-k_{l}\right)\right\rangle
$$

and $T_{p_{i}} \mathbb{F}\left(k_{1}, \ldots, k_{l} ; n\right) \cap T_{\pi^{-1}\left(p_{i}\right)} \mathbb{F}\left(k_{l+1}-k_{l}, \ldots, k_{r}-k_{l} ; n-k_{l}\right)=\emptyset$.

Now, observe that if $T_{\pi^{-1}\left(p_{i}\right)} \mathbb{F}\left(k_{1}, \ldots, k_{r} ; n\right) \cap T_{\pi^{-1}\left(p_{j}\right)} \mathbb{F}\left(k_{1}, \ldots, k_{r} ; n\right) \neq \emptyset$ then

$$
\operatorname{dim}\left\langle T_{\pi^{-1}\left(p_{j}\right)} \mathbb{F}\left(k_{1}, \ldots, k_{r} ; n\right) ; j=1, \ldots, h\right\rangle \leq h \operatorname{dim} \mathbb{F}\left(k_{1}, \ldots, k_{l} ; n\right)+h-2
$$

Since $T_{\pi^{-1}\left(p_{i}\right)} \mathbb{F}\left(k_{l+1}-k_{l}-1, \ldots, k_{r}-k_{l}-1 ; n-k_{l}-1\right)$ is contracted by $\pi$ for any $j=1, \ldots, h$ we have that

$$
\begin{aligned}
\operatorname{dim} \pi(T) & \leq h \operatorname{dim} \mathbb{F}\left(k_{1}, \ldots, k_{r} ; n\right)+h-2-h \operatorname{dim} \mathbb{F}\left(k_{l+1}-k_{l}, \ldots, k_{r}-k_{l} ; n-k_{l}\right) \\
& =h \operatorname{dim} \mathbb{F}\left(k_{1}, \ldots, k_{l} ; n\right)+h-2
\end{aligned}
$$

where $T=\left\langle T_{\pi^{-1}\left(p_{i}\right)} \mathbb{F}\left(k_{1}, \ldots, k_{r} ; n\right) ; i=1, \ldots, h\right\rangle$.

In particular, by Terracini's lemma [Ter12] we have that if $\mathbb{F}\left(k_{1}, \ldots, k_{l} ; n\right)$ is not $h$-defective, then $\mathbb{F}\left(k_{1}, \ldots, k_{r} ; n\right)$ is not $h$-defective.

Theorem 4.14. Consider a flag variety $\mathbb{F}\left(k_{1}, \ldots, k_{r} ; n\right)$ with $n<2 k_{r}+1$. Assume that $n \geq 2 k_{j}+1$ for some index $j$ and let $l$ be the maximum among these $j$ 's. Then, for

$$
h \leq\left(\frac{n+1}{k_{l}+1}\right)^{\left\lfloor\log _{2}\left(\sum_{j=1}^{l} k_{j}+l-1\right)\right\rfloor}
$$

$\mathbb{F}\left(k_{1}, \ldots, k_{r} ; n\right)$ is not $(h+1)$-defective.

Proof. It is an immediate consequence of Theorem 4.11 and Remark 4.13 , 
4.14. On identifiability of products of Grassmannians and flag varieties. Let $X \subset \mathbb{P}^{N}$ be an irreducible non-degenerated variety. A point $p \in \mathbb{P}^{N}$ is said to be $h$-identifiable, with respect to $X$, if it lies on a unique $(h-1)$-plane $h$-secant to $X$. Furthermore, $X$ is said to be $h$-identifiable if a general point of $\operatorname{Sec}_{h}(X)$ is $h$-identifiable.

Now, we combine our bounds on non-secant defectivity of products of Grassmannians and flag varieties and CM19, Theorem 3] to get the following.

Corollary 4.15. Consider the product of Grassmannians $\prod_{i=1}^{r} \mathbb{G}\left(k_{i}, n\right)$. Assume that $2 \prod_{i=1}^{r}\left(k_{i}+1\right)\left(n-k_{i}\right)-$ $1 \leq\left(\frac{n+1}{k_{r}+1}\right)^{\left\lfloor\log _{2}\left(\sum k_{i}+r-1\right)\right\rfloor}$. Then, $\prod_{i=1}^{r} \mathbb{G}\left(k_{i}, n\right)$ is h-identifiable for $h \leq\left(\frac{n+1}{k_{r}+1}\right)^{\left\lfloor\log _{2}\left(\sum k_{i}+r-1\right)\right\rfloor}$.

Furthermore, let us suppose that $n \geq 2 k_{j}+1$ for some index $j$ and consider $l$ the maximum among these $j$ 's. Assume that $2\left(\left(k_{1}+1\right)\left(n-k_{1}\right)+\sum_{j=2}^{i}\left(n-k_{j}\right)\left(k_{j}-k_{j-1}\right)\right)-1 \leq\left(\frac{n+1}{k_{l}+1}\right)^{\left\lfloor\log _{2}\left(\sum_{j=1}^{l} k_{j}+l-1\right)\right\rfloor}$. Then $\mathbb{F}\left(k_{1}, \ldots, k_{r} ; n\right)$ is $h$-identifiable for $h \leq\left(\frac{n+1}{k_{l}+1}\right)^{\left\lfloor\log _{2}\left(\sum_{j=1}^{l} k_{j}+l-1\right)\right\rfloor}$.

Proof. It is enough to apply Corollary 3.4, Theorem 4.14 and [CM19, Theorem 3].

\section{ON THE CHORDAL VARIETY OF $\mathbb{F}(0, k ; n)$}

In this section we consider particularly flag varieties parametrizing chains of type $p \in H^{k} \subset \mathbb{P}^{n}$.

Proposition 5.1. Let us consider the flag variety $\mathbb{F}(0, k ; n) \subset \mathbb{P}(\Gamma) \subset \mathbb{P}^{N}$, where $0<k<n$. Then, $\operatorname{Sec}_{2} \mathbb{F}(0, k ; n)$ has always the expected dimension except when $k=n-1$, in this case $\mathbb{F}(0, n-1 ; n)$ is 2 -defective with 2 -defect $\delta_{2}(\mathbb{F}(0, n-1 ; n))=1$.

Proof. Let $p, q \in \mathbb{F}(0, k ; n)$ be two general points, without lose the generality we can assume that $p=e_{0,\{0, \ldots, k\}}=$ $e_{0, I_{0}}$ and $q=e_{n,\{n-k, \ldots, n\}}=e_{n, I_{1}}$.

Now, Proposition 4.4 yields that

$$
T_{e_{0, I_{0}}} \mathbb{F}(0, k ; n)=\left\langle e_{i, I} \mid d\left((i, I),\left(0, I_{0}\right)\right) \leq 1\right\rangle \cap \mathbb{P}(\Gamma)
$$

and

$$
T_{e_{n, I_{1}}} \mathbb{F}(0, k ; n)=\left\langle e_{i, I} \mid d\left((i, I),\left(n, I_{n}\right)\right) \leq 1\right\rangle \cap \mathbb{P}(\Gamma)
$$

Note that $d\left((i, I),\left(0, I_{0}\right)\right)=1$ if and only if either $i \neq 0$ and $I=I_{0}$ or $i=0$ and $\left|I \cap I_{0}\right|=k$. Similarly, $d\left((i, I),\left(n, I_{1}\right)\right)=1$ if and only if either $i \neq n$ and $I=I_{1}$ or $i=n$ and $\left|I \cap I_{1}\right|=k$. Therefore, since $n \neq 0$ and $I_{1} \neq I_{0}$ we have that $e_{i, I} \in\left\{e_{i, I} \mid d\left((i, I),\left(0, I_{0}\right)\right) \leq 1\right\} \cap\left\{e_{i, I} \mid d\left((i, I),\left(n, I_{1}\right)\right) \leq 1\right\}$ if and only if either $I=I_{0}$ and $i=n$ or $I=I_{1}$ and $i=0$.

Now, assume that $I=I_{0}$ and $i=n$, this is $e_{i, I} \in T_{e_{0, I_{0}}} \mathbb{F}(0, k ; n) \cap T_{e_{n, I_{1}}} \mathbb{F}(0, k ; n)$, in particular we have $\left|I \cap I_{1}\right|=\left|I_{0} \cap I_{1}\right|=k$ and hence $\{1, \ldots, k\} \subset I_{1}$ once $0 \notin I_{1}$. So we must have $k=n-1$. Similarly, if $I=I_{1}$ and $i=0$ we conclude that $k=n-1$.

Therefore, if $k<n-1$, we get

and hence

$$
\left\{e_{i, I} \mid d\left((i, I),\left(0, I_{0}\right)\right) \leq 1\right\} \cap\left\{e_{i, I} \mid d\left((i, I),\left(n, I_{1}\right)\right) \leq 1\right\}=\emptyset
$$

which implies that

$$
\left\{e_{i, I} \mid d\left((i, I),\left(0, I_{0}\right)\right) \leq 1\right\} \cap\left\{e_{i, I} \mid d\left((i, I),\left(n, I_{1}\right)\right) \leq 1\right\} \cap \mathbb{P}(\Gamma)=\emptyset
$$

$$
\operatorname{dim}\left\langle T_{e_{0, I_{0}}} \mathbb{F}(0, k ; n), T_{e_{n, I_{1}}} \mathbb{F}(0, k ; n)\right\rangle=2 \operatorname{dim} \mathbb{F}(0, k ; n)+1
$$

So, Terracini's lemma Ter12 yields that $\operatorname{Sec}_{2} \mathbb{F}(0, k ; n)$ has the expected dimension whenever $k<n-1$.

Now, assume that $k=n-1$. In this case we have

$$
\left\{e_{i, I} \mid d\left((i, I),\left(0, I_{0}\right)\right) \leq 1\right\} \cap\left\{e_{i, I} \mid d\left((i, I),\left(n, I_{1}\right)\right) \leq 1\right\}=\left\{e_{0,\{1, \ldots, n\}}, e_{n,\{0, \ldots, n-1\}}\right\}
$$

Furthermore, $\mathbb{F}(0, n-1 ; n)$ is the hypersurface cutting out in $\mathbb{P}^{n} \times \mathbb{P}^{n *}$ by

$$
\sum_{i=0}^{n}(-1)^{i} Z_{i, I_{n} \backslash\{i\}}=0
$$

where $I_{n}=\{0, \ldots, n\}$.

Therefore, we get that $T_{e_{0, I_{0}}} \mathbb{F}(0, n-1 ; n)=\left\langle e_{i, I} \mid d\left((i, I),\left(0, I_{0}\right)\right) \leq 1\right\rangle \cap \mathbb{P}(\Gamma)$ is given by

$$
\left\langle e_{0,\{1, \ldots, n\}}+(-1)^{n+1} e_{n,\{0, \ldots, n-1\}} ; e_{i, I}\right| d\left((i, I),\left(0, I_{0}\right)\right) \leq 1 \text { and } i, I \neq\left\{\begin{array}{l}
0,\{1, \ldots, n\} \\
n,\{1, \ldots, n-1\}
\end{array}\right\rangle
$$


and $T_{e_{n, I_{1}}} \mathbb{F}(0, n-1 ; n)=\left\langle e_{i, I} \mid d\left((i, I),\left(n, I_{1}\right)\right) \leq 1\right\rangle \cap \mathbb{P}(\Gamma)$ is given by

$$
\left\langle e_{0,\{1, \ldots, n\}}+(-1)^{n+1} e_{n,\{0, \ldots, n-1\}} ; e_{i, I}\right| d\left((i, I),\left(n, I_{1}\right)\right) \leq 1 \text { and } i, I \neq\left\{\begin{array}{l}
0,\{1, \ldots, n\} \\
n,\{1, \ldots, n-1\}
\end{array}\right\rangle
$$

Therefore,

$$
\operatorname{dim}\left\langle T_{e_{0, I_{0}}} \mathbb{F}(0, n-1 ; n), T_{e_{n, I_{1}}} \mathbb{F}(0, n-1 ; n)\right\rangle=2 \operatorname{dim} \mathbb{F}(0, n-1 ; n)<\operatorname{expdim} \operatorname{Sec} \mathbb{F}_{2} \mathbb{F}(0, n-1 ; n)
$$

Finally, since expdim $\operatorname{Sec}_{2} \mathbb{F}(0, k ; n)=2 \operatorname{dim} \mathbb{F}(0, n-1 ; n)+1$ we have that $\mathbb{F}(0, n-1, n)$ is 2-defective with 2-defect $\delta_{2}(\mathbb{F}(0, n-1 ; n))=1$.

\section{REFERENCES}

[AMR19] C. Araujo, A. Massarenti, and R. Rischter, On non-secant defectivity of Segre-Veronese varieties, Transactions of the American Mathematical Society 371 (2019), no. 4, 2255-2278.

[BD10] K. Baur and J. Draisma, Secant dimensions of low-dimensional homogeneous varieties, Adv. Geom. 10 (2010), no. 1, 1-29. MR 2603719

[BK09] W. B. Brett and T. G. Kolda, Tensor decompositions and applications, SIAM Rev. 51 (2009), no. 3, 455-500. MR 2535056

[BL18] J. Brown and V. Lakshmibai, Flag varieties, Texts and Readings in Mathematics, vol. 53, Hindustan Book Agency, Delhi, 2018, An interplay of geometry, combinatorics, and representation theory, Second edition of [ MR2474907]. MR 3890042

[Bri05] M. Brion, Lectures on the geometry of flag varieties, Topics in cohomological studies of algebraic varieties, Trends Math., Birkhäuser, Basel, 2005, pp. 33-85. MR 2143072

[CC02] L. Chiantini and C. Ciliberto, Weakly defective varieties, Trans. Amer. Math. Soc. 354 (2002), no. 1, $151-178$. MR 1859030

[CC10] - On the dimension of secant varieties, J. Eur. Math. Soc. (JEMS) 12 (2010), no. 5, 1267-1291. MR 2677616

[CGLM08] P. Comon, G. Golub, L-H Lim, and B. Mourrain, Symmetric tensors and symmetric tensor rank, SIAM J. Matrix Anal. Appl. 30 (2008), no. 3, 1254-1279. MR 2447451

[CM19] A. Casarotti and M. Mella, From non defectivity to identifiability, https://arxiv.org/abs/1911.00780 2019.

[DL13a] I. Domanov and L. De Lathauwer, On the uniqueness of the canonical polyadic decomposition of third-order tensorsPart I: Basic results and uniqueness of one factor matrix, SIAM J. Matrix Anal. Appl. 34 (2013), no. 3, 855-875. MR 3072760

[DL13b] - On the uniqueness of the canonical polyadic decomposition of third-order tensors-Part II: Uniqueness of the overall decomposition, SIAM J. Matrix Anal. Appl. 34 (2013), no. 3, 876-903. MR 3072761

[DL15] Generic uniqueness conditions for the canonical polyadic decomposition and INDSCAL, SIAM J. Matrix Anal. Appl. 36 (2015), no. 4, 1567-1589. MR 3421620

[Dra08] J. Draisma, A tropical approach to secant dimensions, J. Pure Appl. Algebra 212 (2008), no. 2, 349-363. MR 2357337

[FMR18] A. Barbosa Freire, A. Massarenti, and R. Rischter, Projective aspects of the geometry of lagrangian grassmannians and spinor varieties, https://arxiv.org/abs/1812.07474, 2018.

[GV85] I. Gessel and G. Viennot, Binomial determinants, paths, and hook length formulae, Adv. in Math. 58 (1985), no. 3, 300-321. MR 815360

[KAL11] A. Karfoul, L. Albera, and L. De Lathauwer, Iterative methods for the canonical decomposition of multi-way arrays: Application to blind underdetermined mixture identification, Signal Processing 91 (2011), no. 8, 1789-1802.

[LO15] J. M. Landsberg and G. Ottaviani, New lower bounds for the border rank of matrix multiplication, Theory Comput. 11 (2015), 285-298. MR 3376667

[MR13] A. Massarenti and E. Raviolo, The rank of $n \times n$ matrix multiplication is at least $3 n^{2}-2 \sqrt{2} n^{\frac{3}{2}}-3 n$, Linear Algebra Appl. 438 (2013), no. 11, 4500-4509. MR 3034546

[MR19] A. Massarenti and R. Rischter, Non-secant defectivity via osculating projections, Annali della Scuola Normale Superiore di Pisa, Classe di Scienze, XIX (2019), 1-34.

[Rus03] F. Russo, Tangents and secants of algebraic varieties: notes of a course, Publicações Matemáticas do IMPA. [IMPA Mathematical Publications], Instituto de Matemática Pura e Aplicada (IMPA), Rio de Janeiro, 2003, 24o Colóquio Brasileiro de Matemática. [24th Brazilian Mathematics Colloquium]. MR 2028046

[SB00] N. D. Sidiropoulos and R. Bro, On the uniqueness of multilinear decomposition of $N$-way arrays, Journal of Chemometrics 14 (2000), no. 3, 229-239.

[Ter12] A. Terracini, Sulle $V_{k}$ che rappresentano più di $\frac{k(k-1)}{2}$ equazioni di laplace linearmente indipendenti, Rend. Circ. Mat. Palermo 33 (1912), 176186.

[Tev05] E. A. Tevelev, Projective duality and homogeneous spaces, Encyclopaedia of Mathematical Sciences, vol. 133, SpringerVerlag, Berlin, 2005, Invariant Theory and Algebraic Transformation Groups, IV. MR 2113135 
Ageu Barbosa Freire, Instituto de Matemática e Estatística, Universidade Federal Fluminense, Campus Gragoatá, Rua Alexandre Moura 8 - São Domingos, 24210-200 Niterói, Rio de Janeiro, Brazil

E-mail address: ageufreire@id.uff.br

Alex Casarotti, Dipartimento di Matematica e Informatica, Università di Ferrara, Via Machiavelli 30,44121 FERrara, ItALY

E-mail address: csrlxa@unife.it

Alex Massarenti, Dipartimento di Matematica e Informatica, Università di Ferrara, Via Machiavelli 30,44121 Ferrara, ItaLy

E-mail address: alex.massarenti@unife.it 